Pacific Journal of Mathematics

THUDS IN AFN CONNECTION THE 


\title{
METHODS IN AFFINE CONNECTION THEORY
}

\author{
HARLEY FLANDERS
}

1. Introduction. The paper before us is a continuation of the author's recent work [7] on affinely connected spaces. The content of the paper is best set forth by brief summaries of the various sections. Section 2 gives some properties of the curvature operator $\mathbf{d}^{2}$. In $\S 3$, a contraction operator is introduced into the spaces of $p$-vectors with $q$-form coefficients; and in $\& 4$ this is used to obtain the Ricci tensor in an operator form. In $\S 5$ there is defined an extension of a linear transformation which is analogous to, and consistent with, the corresponding definition for an affine connection; this is shown in $\S 6$, where also certain combinations of connections are discussed. In $\S 7$, a new contraction operator is introduced; this has the advantage of setting up a duality in certain of the linear spaces involved, allowing one to define the adjoint of a linear transformation. This leads to invariant characterizations of symmetric and skew transformations and to decomposition theorems in this and the following $\& 8$. The duality is extended in $\$ 9$, where the adjoint of a connection is defined and relations between it and the connection are given. In $\$ 10$ through 12 , the theory of a series of invariants of a connection introduced by S.S. Chern is developed. After a number of special results, we give a version of an ingenious proof of A. Weil. In the final $\S 13$, we discuss the invariance of the Weyl tensor under projective change of connection and some related tensors which are meaningful for nonsymmetric connections.

2. The linear property of $\Theta$. Let us consider the result

$$
\mathbf{d}^{2 r} \mathbf{e}=\Theta^{r} \mathbf{e}
$$

(7, Formula 9.5), and especially the case $r=1$ :

$$
\mathbf{d}^{2} \mathbf{e}=\Theta \mathbf{e}
$$

From this we deduced that the transformation law for $\Theta$ under a change of frame $\mathbf{e}^{*}=A \mathbf{e}$ is given by

Received October 18, 1953.

Pacific J. Math. 5 (1955), $391-431$ 


$$
\Theta^{*}=A \Theta A^{-1}
$$

(7, Formula 11.5). This means that $\Theta$ transforms as a mixed tensor of order two and hence represents a linear transformation. We can make this precise as follows. Let $\mathbf{v} \in \underset{q}{\mathfrak{J}^{p}}$ so that $\mathbf{d}^{2} \mathbf{v} \in \mathcal{J}_{q+2^{2}}{ }^{2}$ If $f$ is any function, then

$$
\mathbf{d}^{2}(f \mathbf{v})=\mathbf{d}(d f \mathbf{v}+f d \mathbf{v})=d^{2} f \mathbf{v}-d f \mathbf{d} \mathbf{v}+d f \mathbf{d} \mathbf{v}+f \mathbf{d}^{2} \mathbf{v}, \mathbf{d}^{2}(f \mathbf{v})=f \mathbf{d}^{2} \mathbf{v} .
$$

Thus the mapping

$$
\mathbf{d}^{2}: \underset{q}{\not \supset} \rightarrow \underset{q+2}{\not p}
$$

is linear. In the special case $p=1, q=0$, the matrix of $\mathbf{d}^{2}$ with respect to the frame $e$ is exactly $\Theta$. In other cases, the matrix of the operator $d^{2}$ is fairly complicated, but there is no point in writing it down. We remark that the relation

$$
\mathbf{d}^{2} \mathbf{e}=\Theta \mathbf{e}
$$

clearly displays the curvature as a second derivative, which is how it appears in elementary geometry.

If we do not restrict attention to a single space $\zeta_{q}$, we can deduce more than the fact that $\mathbf{d}^{2}$ is linear.

THEOREM 2.1. The operator $\mathbf{d}^{2}$ is linear and is a derivation. This latter

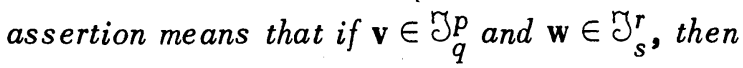

$$
d^{2}(v w)=d^{2} v w+v d^{2} w \text {. }
$$

The necessary computation is easy:

$$
\begin{aligned}
\mathbf{d}^{2}(\mathbf{v w}) & =\mathbf{d}\left[\mathbf{d v} \mathbf{w}+(-1)^{q} \mathbf{v d w}\right] \\
& =\mathbf{d}^{2} \mathbf{v} \mathbf{w}+(-1)^{q+1} \mathbf{d v d w}+(-1)^{q} \mathbf{d v d w}+(-1)^{2 q} \mathbf{v} \mathbf{d}^{2} \mathbf{w} \\
& =\mathbf{d}^{2} \mathbf{v} \mathbf{w}+\mathbf{v} \mathbf{d}^{2} \mathbf{w} .
\end{aligned}
$$

3. Contraction. The space $\gamma_{1}^{0}$ of one-forms is the dual of the space $\mathcal{J}_{0}^{1}$ of one-vectors. As in $[\boldsymbol{\gamma}]$, we shall let $[\omega, \mathbf{v}]$ denote the effect of the form $\omega$ (considered as a functional) on the vector $\mathbf{v}$. Contraction, in tensor analysis, is based on the application of this operation to one covariant and one contravariant 
component of a mixed tensor. Our space $\nearrow_{q}^{p}$ can be considered as the subspace of the space of $p$-contravariant, $q$-covariant tensors whose elements are skew in all contravariant indices and skew in all covariant indices. Having this, we could apply the contraction operator to the elements of $\mathrm{J}_{q}^{p}$ and, with a little modification, obtain the operator that we seek on $\jmath_{q}^{p}$ into $\underset{q-1}{q} \mathcal{J}_{q-1}$. It seems best however to construct this operator directly, within the framework of the spaces we are using.

We begin by looking at the mapping

$$
\begin{aligned}
& \Xi\left(\omega^{\prime}, \cdots, \omega^{q+1} ; \mathbf{v}_{1}, \cdots, \mathbf{v}_{p+1}\right) \\
& =\frac{1}{(p !)(q !)} \sum_{\sigma, \tau} \epsilon_{\sigma} \epsilon_{\tau}\left[\omega^{\tau(q+1)}, \mathbf{v}_{\sigma(p+1)}\right] \omega^{\tau(1)} \cdots \omega^{\tau(q)} \mathbf{v}_{\sigma(1)} \cdots \mathbf{v}_{\sigma(p)},
\end{aligned}
$$

where $\sigma \in S_{p+1}$, the $(p+1)$-symmetric group, $\tau \in S_{q+1}$, and $\epsilon_{\sigma}$ denotes the sign of $\sigma$. The domain and range of $\Xi$ can be indicated as follows:

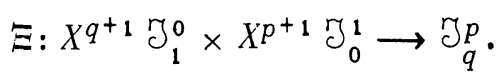

It is evident that $\Xi$ is multilinear and that it is alternating in each set of variables separately. We use the results of Bourbaki [1, Scholia, p. 7 and p. 64] to deduce the existence of a unique linear transformation $\mathbf{C}$,

$$
\mathbf{C}: \underset{q+1}{\gamma_{q}^{+1}} \rightarrow \underset{q}{\jmath_{p}}
$$

such that

$$
\mathbf{C}\left(\omega^{1} \cdots \omega^{q+1} \mathbf{v}_{1} \cdots \mathbf{v}_{p+1}\right)=\Xi\left(\omega^{1}, \cdots, \omega^{q+1} ; \mathbf{v}_{1}, \cdots, \mathbf{v}_{p+1}\right)
$$

It is this $\mathbf{C}$ that we shall call the contraction operator.

If $n$ denotes the dimension of our underlying manifold $M$, then we have

$$
\mathbf{C}(d P)=n
$$

For $d P=\sigma^{i} \mathbf{e}_{i}$ and so

$$
\mathbf{C}(d P)=\sum\left[\sigma^{i}, \mathbf{e}_{i}\right]=\sum 1=n
$$

If $\mathbf{v} \in \mathfrak{J}_{1}^{0}$, the divergence of $\mathbf{v}$ may be defined by 


$$
\operatorname{div}(\mathbf{v})=\mathbf{C}(\mathbf{d v}) .
$$

If we use the notation of $[7, \S 12]$, and if $\mathbf{v}=\lambda^{i} \mathbf{e}_{i}$, then

$$
\operatorname{div}(\mathbf{v})=\lambda^{i}{ }_{i}+\lambda^{i} \Gamma_{i}{ }_{j}^{j}
$$

In fact we have

$$
\begin{aligned}
\operatorname{div}(\mathbf{v}) & =\mathbf{C}(\mathbf{d v})=\mathbf{C}\left(d \lambda^{i} \mathbf{e}_{i}+\lambda^{i} \mathbf{d e}_{i}\right) \\
& =\mathbf{C}\left(\lambda^{i}{ }_{j}{ }{ }^{j} \mathbf{e}_{i}+\lambda^{i} \Gamma_{i}{ }^{j}{ }_{k} \sigma^{k} \mathbf{e}_{i}\right)=\lambda^{i}{ }_{{ }_{i}}+\lambda^{i} \Gamma_{i}{ }^{j}{ }_{j},
\end{aligned}
$$

as asserted.

4. The Ricci tensor. The operator $\mathbf{C d}^{2}$ carries $\mathcal{J}_{0}^{1}$ into $\mathcal{J}_{1}^{0}$. We shall compute its matrix and for this shall need the notation of $[7, \S \S 10,12]$. With $\mathbf{v}=\lambda \mathbf{e}$, we have

$$
\begin{aligned}
& \mathbf{d}^{2} \mathbf{v}=\lambda \Theta \mathbf{e}=\frac{1}{2} \lambda^{i} R_{i}{ }^{j}{ }_{k l} \sigma^{k} \sigma^{l} \mathbf{e}_{j}, \\
& \mathbf{C}\left(\mathbf{d}^{2} \mathbf{v}\right)=\frac{1}{2} \lambda^{i} R_{i}{ }^{j}{ }_{k l}\left\{\left[\sigma^{l}, \mathbf{e}_{j}\right] \sigma^{k}-\left[\sigma^{k}, \mathbf{e}_{j}\right] \sigma^{l}\right\} \\
& \left.=\frac{1}{2} \lambda^{i} R_{i}{ }^{j} k l=\delta_{j}^{l} \sigma^{k}-\delta_{j}^{k} \sigma^{l}\right\} \\
& =\frac{1}{2} \lambda^{i}\left\{R_{i}{ }^{l} k l \sigma^{k}-R_{i}{ }^{k} k l \sigma^{l}\right\}=\lambda^{i} R_{i}{ }^{l} k l{ }^{k} .
\end{aligned}
$$

Thus the matrix is given by $\left\|R_{i k}\right\|$, where $R_{i k}=R_{i}{ }_{k l}$, the known Ricci tensor. We may summarize in the formulas:

$$
\begin{aligned}
& \mathbf{C d}^{2} \mathbf{e}_{i}=R_{i k} \sigma^{k}, \\
& \mathbf{C d}^{2} \mathbf{v}=\lambda^{i} R_{i k} \sigma^{k}, \quad \text { when } \mathbf{v}=\lambda \mathbf{e} .
\end{aligned}
$$

The transformation formula for $R=\left\|R_{i k}\right\|$ follows immediately from the fact that $\mathbf{C d}^{2}$ is linear. If $\mathbf{e}^{*}=A \mathbf{e}$ is a change of frame, then we have

$$
R^{*}=A R^{t} A \text {. }
$$


If $c$ is a closed curve in $M$ which bounds a piece of surface $\Sigma$, then by Stoke's theorem.

$$
\oint_{C} C d^{2} v=\iint_{\Sigma} d\left[C d^{2} v\right] .
$$

It is an interesting problem to characterize by local geometrical conditions when this expression vanishes identically, that is, when $\mathbf{d}\left[\mathbf{C d}^{2} \mathbf{v}\right]=0$. When this is the case, then $\mathbf{C d}^{2} \mathbf{v}$ taken over a chain $\gamma$ is a cocycle.

5. Extension of a linear transformation. An affine connection is, by definition, a certain kind of additive transformation on $\zeta_{0}^{1}$ to $\eta_{1}^{1}$. Given an affine connection d, we showed that it has a natural extension on $\underset{q}{\mathfrak{J}_{p}}$ to $\mathfrak{J}_{q+1}^{p}$. In this section we shall do the same for a linear transformation. Thus we shall deal with the manifold $M$, the derived spaces $\mathcal{J}_{q}$, and shall not assume an affine connection is given.

We suppose given a linear transformation $\mathbf{B}$ on $\mathfrak{J}_{0}^{1}$ to $\mathfrak{J}_{1}^{1}$. Thus

$$
\mathbf{B}(f \mathbf{v}+g \mathbf{w})=f \mathbf{B}(\mathbf{v})+g \mathbf{B}(\mathbf{w})
$$

for $\mathbf{v}, \mathbf{w} \in \mathfrak{J}_{0}^{1}, f$ and $g$ functions. We shall write

$$
\mathbf{B} \in \operatorname{Hom}\left(J_{0}^{1}, \gamma_{1}^{1}\right)
$$

We wish to extend $\mathbf{B}$ to $\mathfrak{J}_{q}{ }_{q}^{p}$. Of course this can be done in several ways, but we shall do it in a manner consistent with the way of extending an affine connection-the precise relationship will be clear in the next section.

First we extend $\mathbf{B}$ to $\mathcal{J}_{0}^{p}$ by the formula

$$
\mathbf{B}\left(\mathbf{v}_{\mathbf{l}} \cdots \mathbf{v}_{p}\right)=\sum \mathbf{v}_{\mathbf{l}} \cdots \mathbf{v}_{j-1}\left(B \mathbf{v}_{j}\right) \mathbf{v}_{j+1} \cdots \mathbf{v}_{p}
$$

where $\mathbf{v}_{1}, \ldots, \mathbf{v}_{p} \in \mathfrak{\jmath}_{0}^{1}$, and by linearity. That this really defines $\mathbf{B}$ on $\jmath_{0}^{p}$ is proved the same way as The orem 7.1 of [7] was.

Next we set $\mathbf{B}(\omega)=0$ for $\omega \in \mathcal{J}_{q}^{0}$. Finally, if $\omega \in T_{q}^{0}$ and $\mathbf{v} \in \mathcal{J}_{0} p$, we set

$$
\mathbf{B}(\omega \mathbf{v})=(-1)^{q} \omega \mathbf{B v} .
$$

Again applying linearity, we have defined $\mathbf{B}$ on $\nearrow_{q}^{p}$. 
THE OREM 5.1. The transformation $\mathbf{B}$ defined above is linear on $\mathfrak{J}_{q}^{p}$ to $\nearrow_{q+1} p$

$$
\mathbf{B} \in \operatorname{Hom}\left(\mathfrak{J}_{q}^{p}, \mathfrak{J}_{q+1}^{p}\right)
$$

If $\mathbf{v} \in \nearrow_{q}^{p}$ and $\mathbf{w} \in \nearrow_{s}^{r}$, then

$$
\mathbf{B}(\mathbf{v w})=(\mathbf{B v}) \mathbf{w}+(-1)^{q} \mathbf{v} \mathbf{B} \mathbf{w} .
$$

It remains to prove the identity (5.4). First let

$$
\mathbf{v}=\mathbf{v}_{\mathbf{1}} \cdots \mathbf{v}_{p}, \quad \mathbf{w}=\mathbf{w}_{1} \cdots \mathbf{w}_{r},
$$

with $\mathbf{v}_{i}, \mathbf{w}_{j}$ in $\breve{J}_{0}$. Then

$$
\begin{aligned}
\mathbf{B}(\mathbf{v} \mathbf{w})= & \mathbf{B}\left(\mathbf{v}_{1}, \cdots, \mathbf{v}_{p} \mathbf{w}_{1} \cdots \mathbf{w}_{r}\right) \\
= & \sum \mathbf{v}_{1} \cdots \mathbf{v}_{i-1}\left(B \mathbf{v}_{i}\right) \mathbf{v}_{i+1} \cdots \mathbf{v}_{p} \mathbf{w}_{1} \cdots \mathbf{w}_{r} \\
& \quad+\sum \mathbf{v}_{1} \cdots \mathbf{v}_{p} \mathbf{w}_{1} \cdots \mathbf{w}_{j-1}\left(B \mathbf{w}_{j}\right) \mathbf{w}_{j+1} \cdots \mathbf{w}_{r}=\mathbf{B}(\mathbf{v}) \mathbf{w}+\mathbf{v} \mathbf{B} .
\end{aligned}
$$

Next, let

$$
\mathbf{v}=\omega \mathbf{v}^{l}, \quad w=\eta \mathbf{w}^{\prime},
$$

where $\omega$ and $\eta$ are $q$ and $s$ forms respectively and $\mathbf{v}^{\prime}$ and $\mathbf{w}^{\prime}$ are $p$ and $r$ vectors, respectively. Then

$$
\begin{aligned}
\mathbf{B}(\mathbf{v} \mathbf{w}) & =\mathbf{B}\left(\omega \eta \mathbf{v}^{\prime} \mathbf{w}^{\prime}\right)=(-1)^{q+s} \omega \eta \mathbf{B}\left(\mathbf{v}^{\prime} \mathbf{w}^{\prime}\right) \\
& =(-1)^{q+s} \omega \eta\left[\mathbf{B}\left(\mathbf{v}^{\prime}\right) \mathbf{w}^{\prime}+\mathbf{v}^{\prime} \mathbf{B} \mathbf{w}^{\prime}\right] \\
& =(-1)^{q} \omega \mathbf{B}\left(\mathbf{v}^{\prime}\right) \eta \mathbf{w}^{\prime}+(-1)^{q} \omega \mathbf{v}^{\prime}(-1)^{s} \eta \mathbf{B} \mathbf{w}^{\prime} \\
& =\mathbf{B}(\mathbf{v}) \mathbf{w}+(-1)^{q} \mathbf{v} \mathbf{B} \mathbf{w} .
\end{aligned}
$$

The general case follows, as usual, by linearity.

6. Algebraic combinations of affine connections. We begin this section with the following result. 
THEORE M 6.1. Let $\mathbf{d}_{\mathbf{1}}$ be an affine connection and $\mathbf{B}$ a linear transformation:

$$
\mathbf{B} \in \operatorname{Hom}\left(\mathfrak{g}_{0}^{1}, g_{1}^{1}\right)
$$

Then the operator $\mathbf{d}_{2}$ defined by

$$
d_{2}=d_{1}+B
$$

is an affine connection. The extension of $\mathbf{d}_{2}$ to $\mathrm{J}_{q}^{p}$ is the sum of the extension of $\mathbf{d}_{1}$ to $\mathrm{Jj}_{q}$ and the extension of $\mathbf{B}$ to $\mathrm{Jj}_{q}$. Thus $(6.1)$ is valid when applied to any of the spaces $\mathrm{Jp}_{q}$.

Proof. We have

$$
\begin{aligned}
& \mathbf{d}_{2}(\mathbf{v}+\mathbf{w})=\mathbf{d}_{2} \mathbf{v}+\mathbf{d}_{2} \mathbf{w} \\
& \mathbf{d}_{2}(f \mathbf{v})=\mathbf{d}_{1}(f \mathbf{v})+\mathbf{B}(f \mathbf{v})=d f \mathbf{v}+f \mathbf{d}_{1} \mathbf{v}+f \mathbf{B v}=d f \mathbf{v}+f \mathbf{d}_{2} \mathbf{v}
\end{aligned}
$$

hence $d_{2}$ is an affine connection.

Next we apply the uniqueness part of Theorem 7.1 of [7]. This asserts that in order to prove that $(6.1)$ is valid when applied to $\underset{q}{\zeta}$, it suffices to show that

$$
\left(\mathbf{d}_{1}+\mathbf{B}\right)(\mathbf{v w})=\left[\left(\mathbf{d}_{\mathbf{1}}+\mathbf{B}\right) \mathbf{v}\right] \mathbf{w}+(-1)^{q} \mathbf{v}\left[\left(\mathbf{d}_{1}+\mathbf{B}\right) \mathbf{w}\right]
$$

for $\mathbf{v} \in \underset{q}{\mathcal{J}_{p}}, \mathbf{w} \in \mathfrak{J}_{s}^{r}$. But this is evidently the case.

It will be convenient to have a space which includes both the affine connections and the linear transformations.

DEFinition 6.1. The space 2 of additive operators consists of all operators $\mathbf{A}$ on $J_{0}^{1}$ to $J_{1}^{1}$ satisfying the single condition

$$
\mathbf{A}(\mathbf{v}+\mathbf{w})=\mathbf{A v}+\mathbf{A w} .
$$

It is clear that $\mathscr{Q}$ is a linear space over the ring $\mathbb{S}(\mathbb{M})$ of infinitely differentiable functions on $M$. Next, we let $\Omega$ denote the space of all linear transforma-

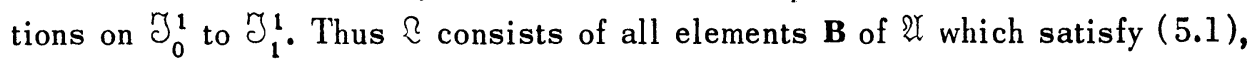
and we see that $\Omega$ is a linear subspace of $\mathscr{U}$. Finally, we denote by $D$ the space of all affine connections. The elements $\mathbf{d}$ of $\mathcal{D}$ satisfy $(6.2)$ and

$$
\mathbf{d}(f \mathbf{v})=d f \mathbf{v}+f \mathbf{d} \mathbf{v} .
$$


THE OREM 6.2. The space 9 is a linear variety of 2 . What is more, ? is a coset of $\Omega$ in $\Re$.

Proof. If $\mathbf{d}_{1}$ is an affine connection and $\mathbf{B} \in \Omega$, then $\mathbf{d}_{\mathbf{1}}+\mathbf{B} \in \mathcal{S}$, by Theorem 6.1. Conversely, if $d_{2} \in \supseteq$, then $B=d_{2}-d_{1} \in \Omega$ since

$$
\begin{aligned}
\mathbf{B}(f \mathbf{v})=\mathbf{d}_{2}(f \mathbf{v})-\mathbf{d}_{\mathbf{1}}(f \mathbf{v}) & =\left(d f \mathbf{v}+f \mathbf{d}_{2} \mathbf{v}\right)-\left(d f \mathbf{v}+f \mathbf{d}_{\mathbf{1}} \mathbf{v}\right) \\
& =f\left(\mathbf{d}_{2} \mathbf{v}-\mathbf{d}_{\mathbf{1}} \mathbf{v}\right)=f \mathbf{B v} .
\end{aligned}
$$

This shows that $\mathscr{D}$ is indeed a coset of $?$.

CoR OLLARY 6.3. If $\mathbf{d}_{1}, \cdots, \mathbf{d}_{r}$ are affine connections, $f^{1}, \ldots, f^{r}$ are functions, and $f^{1}+\cdots+f^{r}=1$, then $\mathbf{d}=f^{1} \mathbf{d}_{1}+\cdots+f^{r} \mathbf{d}_{r}$ is an affine connection.

We can get further results for combinations of connections with constant coefficients. For simplicity, we shall restrict our attention to two summands.

LemMA 6.4. Let $\mathbf{d}_{1}$ and $\mathbf{d}_{2}$ be affine connections,

$$
\mathbf{d}=t_{1} \mathbf{d}_{1}+t_{2} \mathbf{d}_{2},
$$

where $t_{1}$ and $t_{2}$ are constants such that $t_{1}+t_{2}=1$. Then

$$
\begin{aligned}
& \tau=t_{1} \tau_{1}+t_{2} \tau_{2} \\
& \Omega=t_{1} \Omega_{1}+t_{2} \Omega_{2} \\
& \Theta=t_{1} \Theta_{1}+t_{1} t_{2}\left(\Omega_{1}-\Omega_{2}\right)^{2}+t_{2} \Theta_{2} .
\end{aligned}
$$

Here we have used the notation of $[7, \S 8]$. The first formula is a result of the computation

$$
\tau \mathbf{e}=\mathbf{d}(d P)=t_{1} \mathbf{d}_{1}(d P)+t_{2} \mathbf{d}_{2}(d P)=t_{1} \tau_{1} \mathbf{e}+t_{2} \tau_{2} \mathbf{e} .
$$

The second one is obvious, and the third follows from

$$
\begin{aligned}
\Theta & =d \Omega-\Omega^{2}=\left(t_{1} d \Omega_{1}+t_{2} d \Omega_{2}\right)-\left(t_{1}^{2} \Omega_{1}^{2}+t_{1} t_{2} \Omega_{1} \Omega_{2}+t_{1} t_{2} \Omega_{2} \Omega_{1}+t_{2}^{2} \Omega_{2}^{2}\right) \\
& =t_{1}\left(d \Omega_{1}-t_{1} \Omega_{1}^{2}\right)+t_{2}\left(d \Omega_{2}-t_{2} \Omega_{2}^{2}\right)-t_{1} t_{2}\left(\Omega_{1} \Omega_{2}+\Omega_{2} \Omega_{1}\right) \\
& =t_{1}\left(\Theta_{1}+t_{2} \Omega_{1}^{2}\right)+t_{2}\left(\Theta_{2}+t_{1} \Omega_{2}^{2}\right)-t_{1} t_{2}\left(\Omega_{1} \Omega_{2}+\Omega_{2} \Omega_{1}\right) \\
& =t_{1} \Theta_{1}+t_{1} t_{2}\left(\Omega_{1}-\Omega_{2}\right)^{2}+t_{2} \Theta_{2} .
\end{aligned}
$$


This result will be used in $\S 10$, below.

If $\mathbf{d}$ is a fixed affine connection, then as $\mathbf{B}$ runs over all linear transformations, $\mathbf{d}+\mathbf{B}$ runs over all affine connections. This tells us how to construct all of the affine connections on $M$. In effect, assume that $M$ is an $n$-dimensional infinitely differentiable manifold satisfying the second countability axiom. By a theorem of de Rham and Kodaira [5, p. 2 ], there is an open covering $\left\{\mathfrak{U}_{i}\right\}$ of coordinate neighborhoods, and a corresponding partition of unity $\sum \phi_{i}=1$, such that on each $\mathfrak{U}_{i}$, all but a finite number of the $\phi_{j}$ vanish. To construct the most general linear transformation $\mathbf{B}$, we select linear transformations $\mathbf{B}_{i}$ on $\mathfrak{U}_{i}$ (this part is a local problem) and set

$$
\mathbf{B}=\sum \phi_{i} \mathbf{B}_{i}
$$

To construct a single affine connection $\mathbf{d}$ on $M$, we select Riemannian metrics $d s_{i}^{2}$ on $\mathfrak{U}_{i}$ and set

$$
d s^{2}=\sum \phi_{i} d s_{i}^{2}
$$

Then $d s^{2}$ is a metric on $\mathbb{M}$; hence it induces a (symmetric) connection $\mathbf{d}$ on $M$.

We close this section with the following remark. If $d_{0}, d_{1}$ are affine connections, then so is

$$
\mathbf{d}_{t}=t \mathbf{d}_{0}+(1-t) \mathbf{d}_{1}
$$

for $0 \leq t \leq 1$. This linear family of connections suggests the machinery used in the invariance proofs in homology theory. One is led to conjecture that any topological concept which can be defined with respect to a given affine connection will be the same for all affine connections. This opinion is supported by a recent theorem of A. Weil $[4$, p. 57] to be discussed below.

7. Algebraic results. A standard result states that a given connection can be decomposed into the sum of a symmetric connection and a skew-symmetric tensor and that the decomposition is unique. Before analyzing this statement in terms of our calculus, we shall need certain preliminary results.

Let us examine the formulas in [7] which define the torsion tensor. They are (12.3) and (8.4):

$$
\tau^{i}=\frac{1}{2} T_{j k}^{i} \sigma^{j} \sigma^{k}, \quad \text { with } T_{j k}^{i}+T_{k j}^{i}=0
$$




$$
\begin{aligned}
& \mathbf{d}^{2} P=\tau \mathbf{e}, \\
& \tau=d \sigma-\sigma \Omega .
\end{aligned}
$$

In case $\mathbf{e}$ is a local coordinate frame, we have

$$
\sigma^{i}=d u^{i}, \quad d \sigma^{i}=0
$$

and so

$$
\begin{aligned}
& \tau=-\sigma \Omega=\left\|-\sigma^{i} \omega_{i}^{j}\right\|=\left\|-\sigma^{i} \Gamma_{i k}^{j} \sigma^{k}\right\| \\
& T_{j k}^{i}=-\left(\Gamma_{i k}^{j}-\Gamma_{k i}^{j}\right) .
\end{aligned}
$$

Thus in this case the skew-symmetric quantities $\left(\Gamma_{i k}^{j}-\Gamma_{k}{ }_{i}\right)$ are the components of a tensor, while the symmetric quantities

$$
\frac{1}{2}\left(\Gamma_{i k}^{j}+\Gamma_{k i}^{j}\right)=\bar{\Gamma}_{i k}^{j}
$$

are the connection coefficients with respect to the frame $\mathbf{e}$ of a symmetric connection. In the general case, where $e$ is not necessarily a coordinate frame, the term $d \sigma$ is present in $\tau$ and so something more complicated is to be expected.

Let us consider an element $\mathbf{B}$ of

$$
\Omega=\operatorname{Hom}\left(y_{0}^{1}, \eta_{1}^{t}\right)
$$

With respect to a given frame e we may write

$$
\mathbf{B e}_{i}=b_{i k}{ }^{j} \sigma^{k} \mathbf{e}_{j}
$$

If $\mathbf{e}^{*}$ is another frame, then we have

$$
B \mathbf{e}_{i}^{*}=b_{i}^{* j} \sigma^{* k} \mathbf{e}_{j}^{*}
$$

The relation between the frames being $\mathbf{e}_{i}^{*}=a_{i}{ }^{j} \mathbf{e}_{j}$, we shall set

$$
A=\left\|a_{i}^{j}\right\|, \quad C=A^{-1}=\left\|c_{i}^{j}\right\|,
$$

so that 


$$
\sigma^{* i}=\sigma^{j} c_{j}^{i}
$$

and we have

$$
b_{i}^{* j}=a_{i}^{r} a_{k}^{\stackrel{m}{m}} c_{l}^{j} b_{r m}^{l}
$$

It follows that the quantities $h_{i k}{ }^{j}=b_{k}{ }^{j}$ i satisfy the same transformation law and hence the transformation $\mathbf{B}^{*}$ defined by

$$
\mathbf{B}^{*} \mathbf{e}_{i}=b_{k i}^{j}{ }^{k}{ }^{k} \mathbf{e}_{j}
$$

is in ?. We shall call this the adjoint of $\mathbf{B}$.

The purpose of the last paragraph was to motivate the intrinsic procedure which will follow. We begin by introducing a new contraction operator $\mathbf{K}$ defined by:

$$
\begin{aligned}
& K(\mathbf{v}, \mathbf{w})=\mathbf{C}(\mathbf{w}) \mathbf{v}-\mathbf{C}(\mathbf{v w}), \\
& K: \zeta_{0}^{1} \times \zeta_{1}^{1} \rightarrow \zeta_{0}^{1} .
\end{aligned}
$$

I.EMMA 7.1. The mapping $\mathbf{w} \longrightarrow \mathbf{K}(\cdot, \mathbf{w})$ is an isomorphism on $\mathrm{J}_{1}^{1}$ onto End $\left(J_{0}^{1}\right)$, the space of linear transformation on $\zeta_{0}^{1}$ into its elf.

Proof. First we note a formula. If $\mathbf{w}=\omega^{i} \mathbf{v}_{i} \in J_{1}^{1}$ and $\mathbf{v} \in J_{0}^{1}$, then

$$
\begin{aligned}
\mathbf{K}(\mathbf{v}, \mathbf{w}) & =\mathbf{C}(\mathbf{w}) \mathbf{v}-\mathbf{C}(\mathbf{v w})=\mathbf{C}(\mathbf{w}) \mathbf{v}-\mathbf{C}\left(\omega^{i} \mathbf{v} \mathbf{v}_{i}\right) \\
& =\mathbf{C}(\mathbf{w}) \mathbf{v}-\mathbf{C}\left(\omega^{i} \mathbf{v}_{i}\right)+\mathbf{C}\left(\omega^{i} \mathbf{v}\right) \mathbf{v}_{i}=\mathbf{C}\left(\omega^{i} \mathbf{v}\right) \mathbf{v}_{i} .
\end{aligned}
$$

That the mapping of Lemma 7.1 is linear is clear. We shall next prove that it is one-to-one. For suppose $\mathbf{w} \in \mathcal{J}_{1}^{1}$ and $\mathbf{K}(\mathbf{v}, \mathbf{w})=0$ for all $\mathbf{v}$. Then $\left(\omega^{i} \mathbf{v}\right) \mathbf{v}_{i}=0$ for all $\mathbf{v}$. We may assume the $\mathbf{v}_{i}$ are linearly independent and deduce that for each $i, C\left(\omega^{i} \mathbf{v}\right)=0$ for all v. Hence each $\omega^{i}=0, \mathbf{w}=0$. Finally,

$$
\operatorname{dim}\left[\begin{array}{l}
y_{1}^{1} \\
]
\end{array}=\operatorname{dim}\left[\text { End }\left(y_{0}^{1}\right)\right]=n^{2}\right.
$$

and so the mapping must be an isomorphism onto.

THE ORE $\mathrm{M}$ 7.2. Let $\mathbf{B} \in$ ?. Then there exists a unique $\mathbf{B}^{*} \in \Omega$ such that 


$$
\mathbf{K}(\mathbf{v}, \mathbf{B w})=\mathbf{K}\left(\mathbf{w}, \mathbf{B}^{*} \mathbf{v}\right)
$$

for each $\mathbf{v}, \mathbf{w} \in \mathfrak{J}_{0}^{1}$. This defines the adjoint $\mathbf{B}^{*}$ of $\mathbf{B}$. The mapping $\mathbf{B} \longrightarrow \mathbf{B}^{*}$ is an involuntary automorphism of ?.

Proof. Uniqueness is readily proved. If $\mathbf{K}\left(\mathbf{w}, \mathbf{B}^{*} \mathbf{v}\right)=0$ for $\mathbf{v}, \mathbf{w} \in \mathfrak{J}_{0}^{1}$, then by the lemma, $\mathbf{B}^{*} \mathbf{v}=0$ for all $\mathbf{v}$; hence $\mathbf{B}^{*}=0$. To prove existence, let $\mathbf{v}$ be fixed. Then $\mathbf{w} \longrightarrow \mathbf{K}(\mathbf{v}, \mathbf{B} \mathbf{w})$ is an endomorphism of $\mathrm{J}_{0}^{1}$. By the lemma, there is a unique element ( which we denote) $B^{*} \mathbf{v}$ in $\mathcal{J}_{1}^{1}$ such that $K(\mathbf{v}, \mathbf{B} \mathbf{w})=$ $\mathbf{K}\left(\mathbf{w}, \mathbf{B}^{*} \mathbf{v}\right)$ for all $\mathbf{w} \in \mathcal{Y}_{0}^{1}$. It is easily seen that the thereby defined $\mathbf{B}^{*}$ is linear. The rest of the theorem is clear.

We can express this in terms of a frame e. The elements $\sigma^{j} \mathbf{e}_{k}$ form a basis of $y_{1}^{1}$. We find the formulas

$$
\begin{array}{ll}
\mathbf{K}\left(\mathbf{e}_{i}, \sigma^{j} \mathbf{e}_{k}\right)=\delta_{i}{ }^{j} \mathbf{e}_{k}, & \\
\mathbf{K}(\mathbf{v}, \mathbf{w})=\lambda^{i} \mu_{i}{ }^{k} \mathbf{e}_{k}, & \text { for } \mathbf{v}=\lambda^{i} \mathbf{e}_{i}, \mathbf{w}=\mu_{j}{ }^{k} \sigma^{j} \mathbf{e}_{k} .
\end{array}
$$

Suppose $\mathbf{B}$ and $\mathbf{B}^{*}$ are given by

$$
\mathbf{B} \mathbf{e}_{i}=\mathbf{b}_{i k}{ }_{i k} \sigma^{k} \mathbf{e}_{j}, \quad \mathbf{B}^{*} \mathbf{e}_{r}=b_{r}^{*}{ }_{t} \sigma^{t} \mathbf{e}_{s} .
$$

We apply (7.7) to the equality

$$
\mathbf{K}\left(\mathbf{e}_{r}, \mathbf{B} \mathbf{e}_{i}\right)=\mathbf{K}\left(\mathbf{e}_{i}, \mathbf{B}^{*} \mathbf{e}_{r}\right)
$$

to obtain

$$
\begin{aligned}
& b_{i}{ }_{k}^{j} \mathbf{K}\left(\mathbf{e}_{r}, \sigma^{k} \mathbf{e}_{j}\right)=b_{r t}^{* s} \mathbf{k}\left(\mathbf{e}_{i}, \sigma^{t} \mathbf{e}_{s}\right), \\
& b_{i{ }_{k}}^{j} \delta_{r}^{k} \mathbf{e}_{j}=b_{r t}^{* s} \delta_{i}^{t} \mathbf{e}_{s}, \\
& b_{i{ }_{r}}^{j} \mathbf{e}_{j}=b_{r}^{* s} \mathbf{e}_{s}, \\
& b_{i r}^{j}=b_{r i}^{* j} .
\end{aligned}
$$

This is what we anticipated in (7.4).

We shall call $\mathbf{B}$ symmetric if $\mathbf{B}^{*}=\mathbf{B}$ and skew-symmetric if $\mathbf{B}^{*}=-\mathbf{B}$. The 
symmetric transformations $\mathbf{B}$ are characterized by the following theorem.

THEOREM 7.3. The mapping $\mathbf{B} \longrightarrow \mathbf{B}(d P)$ is a linear transformation on

$$
\Omega=\operatorname{Hom}\left(\partial_{0}^{1}, y_{1}^{1}\right)
$$

onto $\mathrm{J}_{2}^{1}$. Its kernel is precisely the space of symmetric elements of $\Omega$. Thus $\mathbf{B}$ is symmetric if and only if $\mathbf{B}(d P)=0$. In general,

$$
\mathbf{B}^{*}(d P)=-\mathbf{B}(d P) \text {. }
$$

Note. The transformation $\mathbf{B}$ is defined on $\mathfrak{J}_{0}^{1}$. When we write $\mathbf{B}(d P)$ we mean the extension of $\mathbf{B}$ given by Theorem 5.1 applied to the displacement vector $d P=\sigma^{i} \mathbf{e}_{i}$ of $\eta_{1}^{1}$.

Proof. It suffices to compute $\mathbf{B}(d P)$ with respect to a frame. Let

$$
\mathbf{B} \mathbf{e}_{i}=b_{i k}{ }_{k} \sigma^{k} \mathbf{e}_{j} \text {. }
$$

By (5.3) we have

$$
\mathbf{B}(d P)=-\sigma^{i} \mathbf{B} \mathbf{e}_{i}=-b_{i k}{ }^{j} \sigma^{i} \sigma^{k} \mathbf{e}_{j}=-\frac{1}{2}\left(b_{i k}{ }_{k}-b_{k}{ }^{j}{ }_{i}\right) \sigma^{i} \sigma^{k} \mathbf{e}_{j} .
$$

From this, everything follows. The result implies that

$$
\operatorname{dim}[\Omega]=\operatorname{dim}\left[r_{2}^{1}\right]+\operatorname{dim}\left[\widetilde{S}_{y}\right]
$$

where $\widetilde{\Xi}_{y}$ denotes the space of symmetric transformations. But this is evident since

$$
\operatorname{dim}[\Omega]=n \cdot n^{2}=n^{3}, \quad \operatorname{dim}\left[\mathcal{J}_{2}^{1}\right]=n\left(\begin{array}{l}
n \\
2
\end{array}\right)=n^{2}(n-1) / 2,
$$

and

$$
\operatorname{dim}\left[\Xi_{y}\right]=n(1+2+\cdots+n)=n^{2}(n+1) / 2 .
$$

Let us agree to denote by $\widetilde{\Xi}_{y}$ the space of symmetric transformations and by $\widetilde{\Xi}_{k}$ the space of skew ones. For the skew transformations we have the following result. 
THEOREM 7.4. B is skew-symmetric if and only if

$$
\mathbf{K}(\mathbf{v}, \mathbf{B v})=0
$$$$
\text { for all } \mathbf{v} \text {. }
$$

This has a familiar proof. If $\mathbf{B}^{*}=-\mathbf{B}$, then

$$
\mathbf{K}(\mathbf{v}, \mathbf{B v})=\mathbf{K}\left(\mathbf{v}, \mathbf{B}^{*} \mathbf{v}\right)=-\mathbf{K}(\mathbf{v}, \mathbf{B v}) ;
$$

hence $\mathbf{K}(\mathbf{v}, \mathbf{B v})=0$. Conversely, if $\mathbf{K}(\mathbf{v}, \mathbf{B v})=0$ for all $\mathbf{v}$, then

$$
\begin{aligned}
& \mathbf{K}(\mathbf{v}+\mathbf{w}, \mathbf{B v}+\mathbf{B w})=0=\mathbf{K}(\mathbf{v}, \mathbf{B w})+\mathbf{K}(\mathbf{w}, \mathbf{B v}) \\
& \mathbf{K}(\mathbf{v}, \mathbf{B w})=-\mathbf{K}(\mathbf{w}, \mathbf{B v})=\mathbf{K}\left(\mathbf{w}, \mathbf{B}^{*} \mathbf{v}\right)
\end{aligned}
$$

hence $\mathbf{B}^{*}=-\mathbf{B}$.

The following is easily derived.

CoOLlaRY 7.5. The spaces $\widetilde{\Xi}_{y}$ and $\widetilde{\Xi}_{k}$ are supplementary in $\Omega$ :

$$
\Omega=\widetilde{\Xi}_{y} \oplus \widetilde{\Xi}_{k} \text {. }
$$

The mapping $\mathbf{B} \rightarrow \mathbf{B}(d P)$ is an isomorphism on $\widetilde{\Xi}_{k}$ onto $\mathfrak{J}_{2}^{1}$.

We note that this is correct from the point of view of dimension since

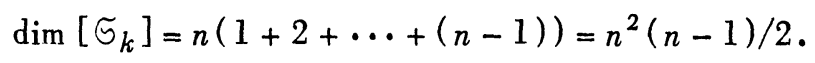

8. Symmetric connections. We recall that a connection $\mathbf{d}$ is called symmetric if

$$
\mathbf{d}^{2} P=\mathbf{d}(d P)=0 \text {. }
$$

We can now state the decomposition theorem referred to at the beginning of the last section.

THEOREM 8.1. If $\mathbf{d}$ is an affine connection, then there exists a unique symmetric connection $\overline{\mathbf{d}}$ and a unique skew-symmetric $\mathbf{B} \in \Omega$ such that

$$
\mathbf{d}=\overline{\mathbf{d}}+\mathbf{B} \text {. }
$$

Proof. If

$$
\overline{\mathbf{d}}+\mathbf{B}=\overline{\mathrm{d}}_{1}+\mathrm{B}_{1},
$$


then

$$
\mathbf{F}=\mathbf{B}_{1}-\mathbf{B}=\overline{\mathbf{d}}-\mathbf{d}_{1}
$$

is symmetric since

$$
\mathbf{F}(d P)=\overline{\mathbf{d}}(d P)-\overline{\mathbf{d}_{1}}(d P)=0,
$$

and skew since

$$
\mathbf{F}^{*}=\mathbf{B}_{1}^{*}-\mathbf{B}^{*}=-\mathbf{B}_{1}+\mathbf{B}=-\mathbf{F}
$$

hence $\mathbf{F}=0$. This proves uniqueness. The decomposition is obtained in this way: If $\mathbf{d}$ is given, then by Corollary 7.1, there is a unique skew-symmetric $\mathbf{B}$ such that

$$
\mathbf{B}(d P)=\mathbf{d}^{2} P \text {. }
$$

Setting $\overline{\mathbf{d}}=\mathbf{d}-\mathbf{B}$, we have

$$
\overline{\mathbf{d}}(d P)=\mathbf{d}^{2} P-\mathbf{d}^{2} P=0
$$

so that $\overline{\mathbf{d}}$ is symmetric.

We shall now express this decomposition in terms of a frame. For this we use the formulas of $[7, \S 12]$. We have

$$
\mathbf{d} \mathbf{e}_{i}=\Gamma_{i k}^{j} \sigma^{k} \mathbf{e}_{j}, \mathbf{d}^{2} P=\tau \mathbf{e}, \tau^{i}=\frac{1}{2} T_{j k}^{i} \sigma^{j} \sigma^{k}
$$

hence

$$
T_{j k}^{i}=h_{j k}^{i}-\left(\Gamma_{j k}^{i}-\Gamma_{k j}^{i}\right)
$$

where

$$
d \sigma^{i}=\frac{1}{2} h_{j k}^{i} \sigma^{j} \sigma^{k}, h_{j k}^{i}+h_{k j}^{i}=0
$$

In the decomposition $\mathbf{d}=\overline{\mathbf{d}}+\mathbf{B}$ we set

$$
\overline{\mathbf{d}} \mathbf{e}_{i}=\bar{\Gamma}_{i}{ }_{k}{ }_{k} \sigma^{k} \mathbf{e}_{j}, \quad \mathbf{B} \mathbf{e}_{i}=b_{i}{ }_{k}{ }_{k} \sigma^{k} \mathbf{e}_{j} .
$$


We require that

$$
\mathbf{B}(d P)=\mathbf{d}^{2} P \quad \text { and } \quad b_{i k}{ }^{j}+b_{k}{ }_{i}{ }_{i}=0 \text {. }
$$

Since

$$
\mathbf{B}(d P)=-\sigma^{j} \mathbf{B} \mathbf{e}_{j}=-\sigma^{j} b_{j k}{ }^{i} \sigma^{k} \mathbf{e}_{i}=-b_{j k}{ }^{i} \sigma^{j} \sigma^{k} \mathbf{e}_{i},
$$

we deduce the relations

$$
\begin{aligned}
& b_{i k}{ }^{j}=-\frac{1}{2} T^{j}{ }_{i k}, \\
& \bar{\Gamma}_{i k}{ }_{k}=\Gamma_{i k}{ }_{k}+\frac{1}{2} T^{j}{ }_{i k}-\frac{1}{2}\left[\left(\Gamma_{i k}{ }_{k}+\Gamma_{k}{ }_{i}{ }\right)+h^{j}{ }_{i k}\right] .
\end{aligned}
$$

We close this section with the following evident result.

THEOREM 8.2. The space $D_{s}$ of symmetric connections is a linear subvariety of the space 9 of all connections. In fact, $\vartheta_{s}$ is a coset in 2 of the space $\widetilde{S}_{y}$ of symmetric transformations.

9. Adjoint of a connection. We have the following result.

THEOREM 9.1. If $\mathbf{d}$ is a given connection, then there exists a unique connection $\mathbf{d}^{*}$, the adjoint of $\mathrm{d}$, such that

(a) $\mathbf{d}^{*}-\mathbf{d}$ is skew,

(b) $\mathbf{d}^{*}(d P)+\mathbf{d}(d P)=0$.

Proof. To prove that $\mathbf{d}^{*}$ exists, we use the decomposition of Theorem 8.1,

$$
\mathbf{d}=\overline{\mathbf{d}}+\mathbf{B},
$$

and set

$$
\mathbf{d}^{*}=\mathbf{d}-\mathbf{B}
$$

to prove that $d^{*}$ is unique, we assume that $d_{1}^{*}$ is another. Then $\left(d_{1}^{*}-d^{*}\right)$ is skew and also symmetric since

$$
\left(\mathbf{d}_{\mathbf{1}}^{*}-\mathbf{d}^{*}\right)(d P)=0
$$


Thus

$$
d_{1}^{*}-d^{*}=0, \quad d_{1}^{*}=d^{*}
$$

On going back to the definition of a skew transformation and using (7.8), we see that either of the following are equivalent to (a) above:

$$
\begin{aligned}
& \left(a^{\prime}\right) \mathbf{K}(\mathbf{v}, \mathbf{d w})+\mathbf{K}(\mathbf{w}, \mathbf{d v})=\mathbf{K}\left(\mathbf{v}, \mathbf{d}^{*} \mathbf{w}\right)+\mathbf{K}\left(\mathbf{w}, \mathbf{d}^{*} \mathbf{v}\right), \\
& \left(a^{\prime \prime}\right) \mathbf{K}(\mathbf{v}, \mathbf{d v})=\mathbf{K}\left(\mathbf{v}, \mathbf{d}^{*} \mathbf{v}\right)
\end{aligned}
$$

We now have the following result.

THEOREM 9.2. The adjoint operation obeys the following rules:

(1) $\left(d^{*}\right)^{*}=\mathbf{d}$.

(2) $\left[t d_{1}+(1-t) d_{2}\right]^{*}=t d_{1}^{*}+(1-t) d_{2}^{*}$.

(3) $\left(d_{1}-d_{2}\right)^{*}=d_{1}^{*}-d_{2}^{*}$.

$A$ connection $\mathbf{d}$ is symmetric if and only if $\mathbf{d}^{*}=\mathbf{d}$.

We shall prove ( 3 ) only, the rest being evident. We set

$$
B=d_{1}-d_{2},
$$

so that $\mathbf{B}$ is a linear transformation and we have

$$
\mathbf{d}_{1}=\mathbf{d}_{2}+\mathbf{B} \text {. }
$$

Then

$$
\begin{aligned}
\mathbf{K}\left(\mathbf{v},\left(\mathbf{d}_{2}^{*}+\mathbf{B}^{*}\right) \mathbf{v}\right) & =\mathbf{K}\left(\mathbf{v}, \mathbf{d}_{2}^{*} \mathbf{v}\right)+\mathbf{K}\left(\mathbf{v}, \mathbf{B}^{*} \mathbf{v}\right) \\
& =\mathbf{K}\left(\mathbf{v}, \mathbf{d}_{2} \mathbf{v}\right)+\mathbf{K}(\mathbf{v}, \mathbf{B v})=\mathbf{K}\left(\mathbf{v}, \mathbf{d}_{2} \mathbf{v}+\mathbf{B} \mathbf{v}\right)=\mathbf{K}\left(\mathbf{v}, \mathbf{d}_{1} \mathbf{v}\right)
\end{aligned}
$$

Since $d_{1}-\left(d_{2}^{*}+B^{*}\right)$ is skew, it follows by Theorem 9.1 that

$$
\mathbf{d}_{1}^{*}=\mathbf{d}_{2}^{*}+\mathbf{B}^{*}
$$

Let us now express the connection coefficients of $\mathbf{d}^{*}$ in terms of those of d. Using the notation of the last section we may write

$$
\mathbf{d}^{*} \mathbf{e}_{i}=\Gamma_{i k}^{* j} \sigma^{k} \mathbf{e}_{j}
$$


and have

$$
\Gamma_{i k}^{* j}=\bar{\Gamma}_{i k}^{j}+b_{i k}^{j}
$$

since $\mathbf{d}^{*}=\overline{\mathbf{d}}-\mathbf{B}$. By $(8.2)$ we have

$$
\Gamma_{i k}^{* j}=\Gamma_{i k}^{j}+T_{i k}^{j}
$$

In the special case of a local coordinate frame

$$
\mathbf{e}_{i}=\partial / \partial u^{i}
$$

we have

$$
\sigma^{i}=d u^{i}, \quad d \sigma^{i}=0,
$$

and

$$
T_{i k}^{j}=-\left(\Gamma_{i k}^{j}-\Gamma_{k}{ }_{i}^{j}\right), \Gamma_{i k}^{* j}=\Gamma_{k}{ }_{i}^{j}
$$

There is another way to prove Theorem 9.1 which, in view of Formula (9.2), gives additional information. We go back to the assertion in Corollary 7.5 that the mapping $\mathbf{B} \longrightarrow \mathbf{B}(d P)$ is an isomorphism on the space of skew-symmetric linear transformations onto $\zeta_{2}^{1}$ - Since $d^{2} P$ is in $\nearrow_{2}^{1}$, it follows that there exists a unique skew transformation $\mathbf{H}$ such that

$$
\mathbf{H}(d P)=-2 \mathbf{d}^{2} P .
$$

We now assert that

$$
\mathbf{d}^{*}=\mathbf{d}+\mathbf{H} \text {. }
$$

For the Conditions (a), (b) of Theorem 9.1 are met since on the one hand

$$
(\mathbf{d}+\mathbf{H})-\mathbf{d}=\mathbf{H}
$$

is skew, and on the other hand by (9.3)

$$
(\mathbf{d}+\mathbf{H})(d P)+\mathbf{d}(d P)=\mathbf{d}^{2} P+\mathbf{H}(d P)+\mathbf{d}^{2} P=0 .
$$




$$
\mathbf{H} \mathbf{e}_{i}=\eta_{i}^{j} \mathbf{e}_{j}
$$

then according to $(9.2)$ we have

$$
\eta_{i}^{j}=T^{j}{ }_{i k} \sigma^{k}, \quad \sigma^{i} \eta_{i}^{j}=2 \tau^{j}
$$

We shall now get some relations between the invariants of $d^{*}$ and those of d. We first may rewrite $(9.6)$ in the form

$$
\sigma H=2 \tau, \text { with } H=\left\|\eta_{i}{ }^{j}\right\|
$$

Thus $H$ is an $n \times n$ matrix of one-forms.

Before proceeding, let us note that the adjoint operation of Theorem 7.2 has a matric analogue. If

$$
K=\left\|\kappa_{i}^{j}\right\|
$$

is an $n \times n$ matrix of one forms, we may set

$$
K^{*}=\left\|\kappa_{i}^{* j}\right\|
$$

where

$$
\kappa_{i}{ }^{j}=K_{i k}{ }^{j} \sigma^{k}, \kappa_{i}^{* j}=K_{i k}^{* j} \sigma^{k}, K_{i k}^{* j}=K_{k i}^{j} .
$$

Having this, we call such a matrix skew if $K^{*}+K=0$, and symmetric if $K^{*}=K$.

We can now say that the matrix $H$ is characterized by the properties (a) $H$ is skew and (b) $\sigma H=2 \tau$. For this is the same as saying that $\mathbf{H}$ is skew and that $(9.3)$ is valid, so Corollary 7.5 applies. Let us denote by $\Omega^{*}, \tau^{*}, \Theta^{*}$, and so on, the quantities associated with $\mathbf{d}^{*}$ which correspond to $\Omega, \tau, \Theta$, and so on. By (9.4) we have

$$
\Omega^{*}=\Omega+H \text {. }
$$

From (9.3) we obtain

$$
\mathbf{d}^{*}(d P)=\mathbf{d}(d P)+\mathbf{H}(d P)=\mathbf{d}(d P)-2 \mathbf{d}^{2} P
$$

hence

$$
\tau^{*}=-\tau
$$


We shall next compute $\Theta *$. We begin with the relation

$$
\mathbf{d}^{*^{2}}=(\mathbf{d}+\mathbf{H})^{2}=\mathbf{d}^{2}+(\mathbf{d H}+\mathbf{H d})+\mathbf{H}^{2} .
$$

Next,

$$
\begin{aligned}
(\mathbf{d H}+\mathbf{H d}) \mathbf{e}= & \mathbf{d}(H \mathbf{e})+\mathbf{H}(\Omega \mathbf{e})=d H \mathbf{e}-H \mathbf{d} \mathbf{e}-\Omega H \mathbf{e} \\
= & (d H-H \Omega-\Omega H) \mathbf{e}, \\
& \mathbf{H}^{2} \mathbf{e}=\mathbf{H}(H \mathbf{e})=-H(\mathbf{H} \mathbf{e})=-H^{2} \mathbf{e} .
\end{aligned}
$$

But

$$
\mathbf{d}^{* 2} \mathbf{e}=\Theta^{*} \mathbf{e} \text { and } \mathbf{d}^{2} \mathbf{e}=\Theta \mathbf{e}
$$

hence

$$
\Theta^{*}=\Theta+d H-H \Omega-\Omega H-H^{2} \text {. }
$$

Now let us differentiate (9.7) and make use of Formulas (8.4) and (8.5) of [7]:

$$
\begin{aligned}
& d \sigma H-\sigma d H=2 d \tau, \\
& (\tau+\sigma \Omega) H-\sigma d H=2(\sigma \Theta-\tau \Omega), \\
& \sigma d H=\sigma \Omega H+\tau H-2 \sigma \Theta+2 \tau \Omega .
\end{aligned}
$$

Multiplying (9.10) by $\sigma$, substituting for $\sigma d H$ this last expression, and using (9.7) yields

$$
\sigma \Theta^{*}=\sigma \Theta+(\sigma \Omega H+\tau H-2 \sigma \Theta+2 \tau \Omega)-\sigma H \Omega-\sigma \Omega H-\sigma H^{2},
$$

(9.12) $\quad \sigma \Theta^{*}=-\sigma \Theta-\tau H$.

Since it may be of interest, we shall also give this relation in tensor notation. Let

$$
\Theta^{*}=\left\|\theta_{i}^{* j}\right\|, \theta_{i}^{* j}=\frac{1}{2} R_{i k l}^{* j} \sigma^{k} \sigma^{l}
$$

Then

(9.13) $R_{i k l}^{* j}+R_{k l i}^{* j}+R_{l}^{* j}{ }_{i k}=-R_{i k l}^{j}-R_{k}{ }_{l i}^{j}-R_{l i k}^{j}-T_{i k}^{r} T_{r l}^{j}-T_{k l}^{r} T_{r i}^{j}-T^{r}{ }_{l i} T_{r k}^{j} \cdot$ 
One easily verifies that differentiation of $(9.10),(9.11)$, or (9.12) yields nothing new.

10. The induced two-form. Given an affine connection d, let us take any moving frame e and consider the matrix $\Theta$ of curvature forms. If $\overline{\mathbf{e}}$ is another frame and if, in their common region of definition, $\mathbf{e}$ and $\overline{\mathbf{e}}$ are related by $\overline{\mathbf{e}}=A \mathbf{e}$, then by $[7, \S 11]$ we have the relation

$$
\bar{\Theta}=A \Theta A^{-1} \text {. }
$$

The elements of the $n \times n$ matrices $\Theta, \bar{\Theta}$ are two-forms and we have as a consequence of $(10.1)$ the relation

$$
S(\bar{\Theta})=S(\Theta),
$$

where $S$ denotes the trace.

DEFINITION 10.1. If $\mathbf{d}$ is an affine connection on $M$, then

$$
\xi(\mathbf{d})=S(\Theta) .
$$

is the induced two-form associated with $\mathbf{d}$. It depends only on d, and not on the particular frame used to define it locally.

THEOREM 10.1. If $\mathbf{d} \mathbf{e}=\Omega \mathbf{e}$, then

$$
\xi(\mathbf{d})=S(d \Omega)=d S(\Omega) .
$$

Consequently $\xi(\mathbf{d})$ is a closed two-form:

$$
d[\xi(\mathbf{d})]=0 .
$$

For $\Theta=d \Omega-\Omega^{2}$; hence

$$
S(\Theta)=S(d \Omega)-S\left(\Omega^{2}\right) .
$$

If $\Omega=\left\|\omega_{i}^{j}\right\|$ as usual, then

$$
S\left(\Omega^{2}\right)=\omega_{i}^{j} \omega_{j}^{i}=-\omega_{j}^{i} \omega_{i}^{j}=-S\left(\Omega^{2}\right) ;
$$

hence $S\left(\Omega^{2}\right)=0$. Also

$$
S(d \Omega)=S\left(\left\|d \omega_{i}^{j}\right\|\right)=d \omega_{i}^{i}=d S(\Omega) .
$$


We next investigate the relation between the induced two-forms for different connections.

It is interesting to observe that the mapping $\xi$ is linear in the following sense.

THE OREM 10.2. If $\mathbf{d}_{1}$ and $\mathbf{d}_{2}$ are connections, and $\mathbf{d}=t_{1} \mathbf{d}_{1}+t_{2} \mathbf{d}_{2}$ for constants $t_{1}$ and $t_{2}$ such that $t_{1}+t_{2}=1$, then

$$
\xi(\mathbf{d})=t_{1} \xi\left(\mathbf{d}_{1}\right)+t_{2} \xi\left(\mathbf{d}_{2}\right)
$$

This follows from Lemma 6.4: since the matrix $\left(\Omega_{1}-\Omega_{2}\right)$ is a matrix of one-forms, the trace of its square vanishes.

THE OREM 10.3. If $\mathbf{d}_{1}, \mathbf{d}_{2}$ are two affine connections on $M$, then there is a one-form $\lambda$ on $M$ such that

$$
\xi\left(\mathbf{d}_{2}\right)-\xi\left(\mathbf{d}_{1}\right)=d \lambda
$$

If $\mathbf{B}$ is the linear transformation $\mathbf{d}_{2}-\mathbf{d}_{1}$, then $\lambda$ may be taken to be the trace $S(\mathbf{B})$, which is the differential one-form defined locally by

$$
\mathbf{B} \mathbf{e}_{i}=\beta_{i}{ }^{j} \mathbf{e}_{j}, S(\mathbf{B})=\beta_{i}{ }^{i}
$$

Consequently $\xi\left(\mathbf{d}_{1}\right)$ and $\xi\left(\mathbf{d}_{2}\right)$ define the same two-dimensional cohomology class.

Proof. Locally, $\Omega_{2}-\Omega_{1}=\left\|\beta_{i}^{j}\right\| ;$ hence

$$
\xi\left(\mathbf{d}_{2}\right)-\xi\left(\mathbf{d}_{1}\right)=d S\left(\Omega_{2}\right)-d S\left(\Omega_{1}\right)=d S\left(\left\|\beta_{i}{ }^{j}\right\|\right)=d \beta_{i}{ }^{i}
$$

It suffices to prove that $\lambda=\beta_{i}{ }^{i}$ is intrinsic. But $\mathbf{B}$ is linear; hence a change of frame $\overline{\mathbf{e}}=A$ e yields

$$
\left\|\bar{\beta}_{i}^{j}\right\|=A\left\|\beta_{i}^{j}\right\| A^{-1} \text { so that } \bar{\beta}_{i}^{i}=\beta_{i}^{i}
$$

We next examine some special cases of this theorem. It is known [6, p. 30] that the most general change of connection which preserves parallelism is given by

$$
\mathbf{d}_{2} \mathbf{v}=\mathbf{d}_{1} \mathbf{v}+\sigma_{0} \mathbf{v}
$$

where $\sigma_{0}$ is a fixed one-form. We may write $\sigma_{0}=\sigma \psi$ with $\sigma=\left(\sigma^{1}, \cdots, \sigma^{n}\right)$ as 
usual, and $\psi={ }^{t}\left(\psi_{1}, \cdots, \psi_{n}\right)$ for functions $\psi_{j}$. We define $\mathbf{H} \in \Omega$ as follows:

$$
\mathbf{H} \mathbf{v}=\sigma_{0} \mathbf{v} \text {. }
$$

Our change of connection is given by the equations

$$
\mathbf{d}_{2}=\mathbf{d}_{1}+\mathbf{H}, \quad \Omega_{2}=\Omega_{1}+\sigma_{0} I,
$$

where $I$ denotes the $n$-rowed identity matrix. We deduce

$$
d \Omega_{2}=d \Omega_{1}+d \sigma_{0} l, \Omega_{2}^{2}=\Omega_{1}^{2}+\sigma_{0} \Omega_{1}+\Omega_{1} \sigma_{0}+\sigma_{0}^{2} l=\Omega_{1}^{2} ;
$$

hence

$$
\Theta_{2}=\Theta_{1}+d \sigma_{0} l, \quad d \Theta_{2}=d \Theta_{1} .
$$

Consequently if $\sigma_{0}$ is closed then $\Theta_{2}=\Theta_{1}$.

Lemma 10.4. The adjoint $\mathbf{H}^{*}$ of $\mathbf{H}$ is given by

$$
\mathbf{H}^{*} \mathbf{v}=\mathbf{C}\left(\sigma_{0} \mathbf{v}\right) d P .
$$

For we have

$$
\begin{aligned}
\mathbf{K}(\mathbf{v}, \mathbf{H} \mathbf{w})=\mathbf{K}\left(\mathbf{v}, \sigma_{0} \mathbf{w}\right)=\mathbf{C}\left(\sigma_{0} \mathbf{v}\right) \mathbf{w} & =\mathbf{C}\left(\sigma_{0} \mathbf{v}\right) \mathbf{K}(\mathbf{w}, d P) \\
& =\mathbf{K}\left(\mathbf{w}, \mathbf{C}\left(\sigma_{0} \mathbf{v}\right) d P\right) .
\end{aligned}
$$

THEOREM 10.5. Consider each of the changes of connection

$$
\text { (a) } \mathbf{d}_{2}=\mathbf{d}_{1}+\mathbf{H}, \quad \text { (b) } \mathbf{d}_{2}=\mathbf{d}_{1}+\mathrm{H}^{*} \text {, }
$$

where $\mathbf{H} \mathbf{v}=\sigma_{0} \mathbf{v}$ for/ a fixed one-form $\sigma_{0}$. Corresponding to these we then have

$$
\left(\mathrm{a}^{\prime}\right) \quad \xi\left(\mathbf{d}_{2}\right)=\xi\left(\mathbf{d}_{1}\right)+n d \sigma_{0}, \quad\left(\mathrm{~b}^{\prime}\right) \quad \xi\left(\mathbf{d}_{2}\right)=\xi\left(\mathbf{d}_{1}\right)+d \sigma_{0} .
$$

The proof is based on Theorem 10.3. For the change (a) the result is evident by (10.11). To prove (b) we observe that

$$
\Omega_{2}=\Omega_{1}+\psi \sigma
$$

when $\sigma_{0}=\sigma \psi$ as above. Hence

$$
S(\psi \sigma)=\psi_{i} \sigma^{i}=\sigma_{0}, \quad \xi\left(\mathbf{d}_{2}\right)=\xi\left(\mathbf{d}_{1}\right)+d \sigma_{0}
$$


Now let us go back to the linear transformation $\mathbf{C} \mathbf{d}^{2}$ of $\$ 4$ which served to define the Ricci tensor:

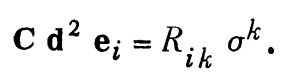

Using the ideas in $\$ 5$ on extending a linear transformation to the various spaces $\mathfrak{J}_{q}$, we shall apply $\mathbf{C} \mathbf{d}^{2}$ to the element $d P$ of $\mathfrak{J}_{1}^{1}$. (Note that

$$
\mathbf{C} \mathbf{d}^{2}: \mathrm{J}_{0}^{1} \rightarrow \mathrm{J}_{1}^{0}
$$

whereas in $\S 5$ we only considered B: $\nearrow_{0}^{1} \longrightarrow \nearrow_{1}^{1}$; consequently we are not applying the results of that section now, but are only drawing an analogy.) We have

$$
\begin{aligned}
\mathbf{C d}^{2}(d P) & =\mathbf{C} \mathbf{d}^{2}\left(\sigma^{i} \mathbf{e}_{i}\right)=-\sigma^{i} \mathbf{C} \mathbf{d}^{2}\left(\mathbf{e}_{i}\right) \\
& =-\sigma^{i} R_{i k} \sigma^{k}=-\frac{1}{2}\left(R_{i k}-R_{k i}\right) \sigma^{i} \sigma^{k} .
\end{aligned}
$$

Thus by skew-symmetrizing the Ricci tensor we obtain another two-form invariantly associated with the connection d. Unfortunately, this is usually not a closed two form. However we do have the following result (cf. [6, p.9]).

THE ORE M 10.6. If $\mathbf{d}$ is a symmetric connection, then

$$
\xi(\mathbf{d})=-\frac{1}{2}\left(R_{i k}-R_{k i}\right) \sigma^{i} \sigma^{k}
$$

Proof. Since $\mathbf{d}$ is symmetric we have $\tau=0, \sigma \Theta=0[7,(8.7)$.$] Thus$

$$
\sigma^{i} \theta_{i}^{j}=0, \quad R_{i k l}^{j} \sigma^{i} \sigma^{k} \sigma^{l}=0, \quad R_{i k l}^{j}+R_{k}{ }^{j}{ }_{l i}+R_{l i k}^{j}=0 .
$$

We contract on $j$ and $l$ to obtain

$$
R_{i k}-R_{k i}+R_{l i k}^{l}=0
$$

But

$$
S(\Theta)=\theta_{l}^{l}=\frac{1}{2} R_{l}{ }_{l i k} \sigma^{i} \sigma^{k}
$$


hence

$$
\left(R_{i k}-R_{k i}\right) \sigma^{i} \sigma^{k}+2 S(\Theta)=0 .
$$

It is known [6, p. 31] that the most general change of connection which preserves the paths of symmetric connections is the so-called projective change of connection [6, p.87] defined by

$$
\overline{\mathbf{d}}=\mathbf{d}+\mathbf{H}+\mathbf{H}^{*}, \quad \mathbf{H} \mathbf{v}=\sigma_{0} \mathbf{v},
$$

for a fixed one-form $\sigma_{0}$. Since $\left(\mathbf{H}+\mathbf{H}^{*}\right)$ is a symmetric linear transformation, this sends a symmetric connection into another such. It is an immediate consequence of Theorem 10.3 that in case of $(10.16)$ we have

$$
\xi(\overline{\mathbf{d}})=\xi(\mathbf{d})+(n+1) d \sigma_{0}
$$

Still one more remark in this connection. If we set $\mathbf{B}=\mathbf{H}+\mathbf{H}^{*}$, then $\mathbf{B}$ is symmetric and

$$
\mathbf{B} \mathbf{v}=\sigma_{0} \mathbf{v}+\mathbf{C}\left(\sigma_{0} \mathbf{v}\right) d P,
$$

so that

$$
\begin{aligned}
\mathbf{B}^{2} \mathbf{v}=\mathbf{B}\left(\sigma_{0} \mathbf{v}+\mathbf{C}\left(\sigma_{0} \mathbf{v}\right) d P\right) & =-\sigma_{0} \mathbf{B v}+\mathbf{C}\left(\sigma_{0} \mathbf{v}\right) \mathbf{B}(d P) \\
& =-\sigma_{0} \mathbf{B v}=-\sigma_{0} \mathbf{C}\left(\sigma_{0} \mathbf{v}\right) d P ;
\end{aligned}
$$

hence

$$
\mathbf{B}^{2} \mathbf{v}=-C\left(\sigma_{0} \mathbf{v}\right) \sigma_{0} d P
$$

If $d s^{2}$ is a metric on $m$ and $\mathbf{d}$ is the induced affine connection, then $\mathbf{d}$ is symmetric and the corresponding Ricci tensor is symmetric. This implies the following:

THEOREM 10.7. If $\mathbf{d}$ is the connection induced by a Riemannian $d s^{2}$, then $\xi(\mathbf{d})=0$.

In view of this last result, The orem 10.3, and our remarks at the end of $\S 6$, we have the corollary:

THE ORE M 10.8. If $\mathbf{d}$ is any affine connection on $M$, then $\xi(\mathbf{d})$ is an exact 
differential form; in other words, $\xi(\mathbf{d})$ is the trivial two-dimensional cohomology class.

Chern $[2,3]$ proves this result by showing that the periods of $\xi(d)$ all vanish, but his proof requires orientability. It is interesting that the essential formulas are substantially on page 9 of $[6]$.

11. Higher dimensional cases. We now form $\Theta^{r}$, the $r$ th power of the curvature matrix, and consider its trace

$$
\xi_{r}=\xi_{r}(\mathbf{d})=S\left(\Theta^{r}\right)
$$

By virtue of (10.1), this defines a $2 r$-form on $\Re$. Chern [2] conjectured that this is a cohomology class and yields a topological invariant of $\mathbb{M}$ alone. In this section we shall obtain partial results towards this conjecture.

First we ned a few elementary results on products.

Lemma 11.1. If $A=\left\|\alpha_{i}{ }^{j}\right\|$ is a matrix of $p$-forms and $B=\left\|\beta_{j}{ }^{k}\right\|$ is a matrix of $q$-forms, then

$$
S(A B)=(-1)^{p q} S(B A) .
$$

For $S(A B)=\alpha_{i}{ }^{j} \beta_{j}{ }^{i}=(-1)^{p q}{\beta_{j}}^{i} \alpha_{i}{ }^{j}=(-1)^{p q} S(B A)$.

COROLLARY. If $A B=B A$ and $p \equiv q \equiv 1(\bmod 2)$, then $S(A B)=0$.

Lemma 11.2. If $A_{1}, \cdots, A_{r}$ are matrices of forms of degrees $p_{1}, \cdots, p_{r}$, respectively, then

$$
{ }^{t}\left(A_{1}, \cdots, A_{r}\right)=(-1)^{c}{ }^{t} A_{r}, \ldots,{ }^{t} A_{2}{ }^{t} A_{1},
$$

where

$$
c=\sum_{1 \leq i<j \leq r} p_{i} p_{j}
$$

This is easily checked by induction. In particular we have the following:

COROLLARY. If $A_{1}, \cdots, A_{r}$ are matrices of one-forms, then

$$
{ }^{t}\left(A_{1}, \cdots, A_{r}\right)=\epsilon_{r}{ }^{t} A_{r}, \cdots,{ }^{t} A_{1},
$$


where $\epsilon_{r}=+1$ if $r \equiv 0,1(\bmod 4)$ and $\epsilon_{r}=-1$ if $r \equiv 2,3(\bmod 4)$.

In [7], we proved the formula (9.1):

$$
d \Theta^{r}=\Omega \Theta^{r}-\Theta^{r} \Omega,
$$

which is a generalization of the Bianchi identity. From this we deduce that $\xi_{r}$ is indeed a cohomology class. In fact,

$$
\begin{aligned}
d \xi_{r} & =d S\left(\Theta^{r}\right)=S\left(d \Theta^{r}\right)=S\left[\Omega \Theta^{r}-\Theta^{r} \Omega\right] \\
& =S\left(\Omega \Theta^{r}\right)-S\left(\Theta^{r} \Omega\right)=S\left(\Omega \Theta^{r}\right)-S\left(\Omega \Theta^{r}\right)=0 .
\end{aligned}
$$

THEOREM 11.3. The 2r-form $\xi_{r}$ is closed and hence defines a cohomology class on $\mathbb{M}$.

In the remainder of this section we shall concentrate on the case $r=2$. We shall begin by expressing $\xi_{2}$ locally as an exterior derivative-thus obtaining a new proof of the foregoing theorem for this special case. In the computations which follow we shall make free use of Lemma 11.1:

$$
\begin{aligned}
\Theta & =d \Omega-\Omega^{2}, \\
\Theta^{2} & =d \Omega d \Omega-d \Omega \Omega^{2}-\Omega^{2} d \Omega+\Omega^{4} \\
S\left(\Omega^{2}\right) & =S\left(d \Omega d \Omega-2 d \Omega \Omega^{2}\right) .
\end{aligned}
$$

But

$$
\begin{aligned}
d\left(\Omega^{3}\right) & =d \Omega \Omega^{2}-\Omega d \Omega \Omega+\Omega^{2} d \Omega, \\
S\left[d \Omega^{3}\right] & =3 S\left[d \Omega \Omega^{2}\right],
\end{aligned}
$$

and

$$
d(d \Omega \Omega)=d \Omega d \Omega
$$

hence

$$
S\left(\Theta^{2}\right)=S\left[d(d \Omega \Omega)-(2 / 3) d \Omega^{3}\right]=d S\left[\Omega d \Omega-(2 / 3) \Omega^{3}\right] \text {. }
$$




$$
\xi_{2}=d S \Phi_{2}
$$

where

$$
\Phi_{2}=d \Omega \Omega-(2 / 3) \Omega^{3} \text {. }
$$

Now we shall compare results for two connections $\mathbf{d}$ and $\overline{\mathbf{d}}$. We write $\overline{\mathbf{d}}=\mathbf{d}+\mathbf{B}$ defining the linear transformation B. We write

$$
\overline{\mathbf{d}} \mathbf{e}=\bar{\Omega} \mathbf{e}, \mathbf{d e}=\Omega \mathbf{e}, \mathbf{B e}=B \mathbf{e},
$$

with $B=\left\|\beta_{i}{ }^{j}\right\|$ a matrix of one-forms. Thus

$$
\bar{\Omega}=\Omega+B .
$$

We have

$$
\begin{gathered}
\bar{\Phi}_{2}=d \bar{\Omega} \bar{\Omega}-(2 / 3) \bar{\Omega}^{3}=(d \Omega+d B)(\Omega+B)-(2 / 3)(\Omega+B)^{3} \\
=(d \Omega \Omega+d \Omega B)+d B \Omega+d B B)-(2 / 3)\left(\Omega^{3}+\Omega^{2} B+\Omega B \Omega\right. \\
\left.+\Omega B^{2}+B \Omega^{2}+B \Omega B+B^{2} \Omega+B^{3}\right), \\
S\left(\bar{\Phi}_{2}\right)=S\left(\Phi_{2}\right)+S\left(d B B-(2 / 3) B^{3}+d \Omega B+d B \Omega-2 B \Omega^{2}-2 \Omega B^{2}\right) .
\end{gathered}
$$

We make the substitution

$$
d B \Omega=B d \Omega-d(B \Omega)
$$

in this to obtain

$$
S\left(\bar{\Phi}_{2}\right)=S\left(\Phi_{2}\right)+S\left(d B B-(2 / 3) B^{3}+2 d \Omega B-d(B \Omega)-2 B \Omega^{2}-2 \Omega B^{2}\right) .
$$

By differentiating we arrive at:

LEMMA 11.5. Locally we have

$$
\xi_{2}(\overline{\mathbf{d}})=\xi_{2}(\mathbf{d})+d S \Psi_{2} .
$$

where

$$
\Psi_{2}=(d B-B \Omega) B-(2 / 3) B^{3}+2 \Theta B-\Omega B^{2} .
$$


It is to be emphasized that in these formulas, although we deal with several connections, everything is expressed in terms of one moving frame e. We shall now investigate what happens under a change of frame,

$$
\hat{\mathbf{e}}=A \mathbf{e} .
$$

From Formula (11.4) of [7] we have

$$
\hat{\Omega}=A \Omega A^{-1}+d A A^{-1}
$$

and from the fact that $\mathbf{B}$ is linear,

$$
\hat{B}=A B A^{-1} \text {. }
$$

Since $\mathbf{d}^{2}$ is linear,

$$
\hat{\Theta}=A \Theta A^{-1} \text {. }
$$

Thus

$$
\begin{aligned}
\hat{\Psi}_{2}= & (d \hat{B}-\hat{B} \hat{\Omega}) \hat{B}-(2 / 3) \hat{B}^{3}+2 \hat{\Theta} \hat{B}-\hat{\Omega} \hat{B}^{2} \\
= & \left(d A B A^{-1}+A d B A^{-1}+A B A^{-1} d A A^{-1}-A B \Omega A^{-1}-A B A^{-1} d A A^{-1}\right) A B A^{-1} \\
& \quad-(2 / 3) A B^{3} A^{-1}+2 A \Theta B A^{-1}-A \Omega B^{2} A^{-1}-d A B^{2} A^{-1} \\
= & A d B B A^{-1}-A B \Omega B A^{-1}-(2 / 3) A B^{3} A^{-1}+2 A \Theta B A^{-1}-A \Omega B^{2} A^{-1} \\
= & A\left[(d B-B \Omega) B-(2 / 3) B^{3}+2 \Theta B-\Omega B^{2}\right] A^{-1}=A \Psi_{2} A^{-1} .
\end{aligned}
$$

It follows that $\Psi_{2}$ represents a linear transformation on $r_{0}^{1}$ to $r_{3}^{1}$. To see this directly we observe that

$$
\begin{aligned}
& \mathbf{d e}=\Omega \mathbf{e}, \mathbf{B d e}=-\Omega B \mathbf{e}, \mathbf{B}^{2} \mathbf{d e}=-\Omega B^{2} \mathbf{e}, \\
& \mathbf{B e}=B \mathbf{e}, \mathbf{d B e}=(d B-B \Omega) \mathbf{e}, \mathbf{B d B e}=(d B-B \Omega) B \mathbf{e} ;
\end{aligned}
$$

hence

$$
\Psi_{2} \mathbf{e}=\left(\mathbf{B d B}+\mathbf{B}^{2} \mathbf{d}+(2 / 3) \mathbf{B}^{3}+2 \mathbf{B d}^{2}\right) \mathbf{e} .
$$

It is clear that $\mathbf{B}^{3}$ and $\mathbf{B} \mathbf{d}^{2}$ are linear since $\mathbf{B}$ and $\mathbf{d}^{2}$ are so (see $\left.\S 2\right)$; 
consequently it remains to verify that

$$
B d B+B^{2} \mathbf{d}=\mathbf{B}(\mathbf{d B}+\mathbf{B d})
$$

is linear, and obviously it suffices to show that the (Jordan) product $\mathbf{d B}+\mathbf{B d}$ is itself linear. As usual we let $f$ be a function, $\mathbf{v} \in \mathcal{J}_{0}^{1}$, and compute:

$$
\begin{aligned}
(\mathbf{d B}+\mathbf{B d})(f \mathbf{v}) & =\mathbf{d}(f \mathbf{B v})+\mathbf{B}(d f \mathbf{v}+f \mathbf{d v}) \\
& =d f \mathbf{B v} f \mathbf{d B v}-d f \mathbf{B v}+f \mathbf{B d v}=f(\mathbf{d B}+\mathbf{B d}) \mathbf{v},
\end{aligned}
$$

exactly what is needed.

As a result of this we have proved:

Lemma 11.6. If $\mathbf{e}$ and $\hat{\mathbf{e}}$ are two frames related by $\hat{\mathbf{e}}=A \mathbf{e}$, then

$$
\hat{\Psi}_{2}=A \Psi_{2} A^{-1} \text {. }
$$

THE OREM 11.7. If $\mathbf{d}$ and $\overline{\mathbf{d}}$ are two affine connections on $M$, then $\xi_{2}(\mathbf{d})$ and $\xi_{2}(\overline{\mathbf{d}})$ define the same 4-dimensional cohomology class on $\mathbb{M}$. More precisely, there is a three-form $\lambda_{2}$ on $M$ such that

$$
\xi_{2}(\overline{\mathbf{d}})=\xi_{2}(\mathbf{d})+d \lambda_{2} .
$$

We merely set $\lambda_{2}=S\left(\Psi_{2}\right)$. By Lemma 11.6, this is intrinsic and so the theorem follows from Lemma 11.5.

Let us return to the case of a single connection d. It is interesting to see how the matrix $\Phi_{2}$ of Lemma 11.4 and its trace transform under change of frame. As above, we let $\hat{\mathbf{e}}=A \mathbf{e}$ and have

$$
\begin{aligned}
\hat{\Phi}_{2}= & d \hat{\Omega} \hat{\Omega}-(2 / 3) \hat{\Omega}^{3} \\
= & \left(d A \Omega A^{-1}+A d \Omega A^{-1}+A \Omega A^{-1} d A A^{-1}+d A A^{-1} d A A^{-1}\right)\left(A \Omega A^{-1}+d A A^{-1}\right) \\
& \quad-(2 / 3)\left(A \Omega A^{-1}+d A A^{-1}\right)^{3} \\
= & {\left[d A \Omega^{2} A^{-1}+A d \Omega \Omega A^{-1}+A \Omega A^{-1} d A \Omega A^{-1}+d A A^{-1} d A \Omega A^{-1}\right.} \\
& \left.\quad+d A \Omega A^{-1} d A A^{-1}+A d \Omega A^{-1} d A A^{-1}+A \Omega A^{-1} d A A^{-1} d A A^{-1}+\left(d A A^{-1}\right)^{3}\right]
\end{aligned}
$$




$$
\begin{gathered}
-(2 / 3)\left[A \Omega^{3} A^{-1}+A \Omega A^{-1} d A \Omega A^{-1}+d A \Omega^{2} A^{-1}+d A A^{-1} d A \Omega A^{-1}\right. \\
\left.+A \Omega^{2} A^{-1} d A A^{-1}+A \Omega A^{-1} d A A^{-1} d A A^{-1}+d A \Omega A^{-1} d A A^{-1}+\left(d A A^{-1}\right)^{3}\right], \\
S\left(\hat{\Phi}_{2}\right)=S\left[2 d A \Omega^{2} A^{-1}+d \Omega \Omega+3 \Omega A^{-1} d A A^{-1} d A+d \Omega A^{-1} d A+\left(d A A^{-1}\right)^{3}\right] \\
-(2 / 3) S\left[\Omega^{3}+3 \Omega^{2} A^{-1} d A+3 d A A^{-1} d A \Omega A^{-1}+\left(d A A^{-1}\right)^{3}\right] ;
\end{gathered}
$$

hence

$$
S\left(\hat{\Phi}_{2}\right)=S\left(\Phi_{2}\right)+S\left[\Omega A^{-1} d A A^{-1} d A+d A \Omega A^{-1}+(1 / 3)\left(d A A^{-1}\right)^{3}\right]
$$

Theorem 11.7 is Chern's conjecture for the case $r=2$. It says in effect that $\xi_{2}$ is a 4-dimensional cohomology class dependent only on the differentiable structure of $M$. To compute it for a given differentiable structure, it suffices to compute it with respect to a suitable Riemannian $d s^{2}$. Lemma 11.2 may be of help in this. Evidently, if $M$ admits a locally euclidean metric, then $\xi_{2}$ is trivial; this is also the case for the 4-sphere. However in general $\xi_{2}$ is not trivial. ${ }^{1}$ Also, we may point out that it is not clear that if the same topological manifold is endowed with two inequivalent differentiable structures, then the same cohomology class will result.

We shall now give some formulas for the cases $r=3$ and $r=4$. To begin with we have

$$
\begin{aligned}
\Theta^{3}=(d \Omega)^{3} & -\Omega^{2}(d \Omega)^{2}-d \Omega \Omega^{2} d \Omega+\Omega^{4} d \Omega \\
& -(d \Omega)^{2} \Omega^{2}+\Omega^{2} d \Omega \Omega^{2}+d \Omega \Omega^{4}-\Omega^{6}, \\
\xi_{3}=S\left(\Theta^{3}\right)= & S\left[(d \Omega)^{3}-3(d \Omega)^{2} \Omega^{2}+3 d \Omega \Omega^{4}\right] .
\end{aligned}
$$

Next we have

$$
\begin{aligned}
& d\left[(d \Omega)^{2} \Omega\right]=(d \Omega)^{3}, \\
& d\left[d \Omega \Omega^{3}\right]=(d \Omega)^{2} \Omega^{2}-d \Omega \Omega d \Omega \Omega+d \Omega \Omega^{2} d \Omega, \\
& d\left[\Omega^{5}\right]=d \Omega \Omega^{4}-\Omega d \Omega \Omega^{3}+\cdots+\Omega^{4} d \Omega ;
\end{aligned}
$$

\footnotetext{
${ }^{1}$ See [8] where this is shown for the case of the complex projective plane by employing its elliptic metric. We are grateful to the referee for this reference.
} 
hence

$$
\begin{aligned}
& d S\left[(d \Omega)^{2} \Omega\right]=S\left[(d \Omega)^{3}\right], \\
& d S\left[d \Omega \Omega^{3}\right]=2 S\left[(d \Omega)^{2} \Omega^{2}\right], \\
& d S\left[\Omega^{5}\right]=5 S\left[d \Omega \Omega^{4}\right] .
\end{aligned}
$$

It follows that

(11.3)

$$
\xi_{3}=d S\left(\Phi_{3}\right),
$$

where

$$
\Phi_{3}=(d \Omega)^{2} \Omega-(3 / 2) d \Omega \Omega^{3}+(3 / 5) \Omega^{5} .
$$

Next we have

$$
\begin{gathered}
\Theta^{4}=(d \Omega)^{4}-\Omega^{2}(d \Omega)^{3}-d \Omega \Omega^{2}(d \Omega)^{2}+\Omega^{4}(d \Omega)^{2}-(d \Omega)^{2} \Omega^{2} d \Omega \\
+\Omega^{2} d \Omega \Omega^{2} d \Omega+d \Omega \Omega^{4} d \Omega-\Omega^{6} d \Omega \\
-(d \Omega)^{3} \Omega^{2}+\Omega^{2}(d \Omega)^{2} \Omega^{2}+d \Omega \Omega^{2} d \Omega \Omega^{2}-\Omega^{4} d \Omega \Omega^{2}+(d \Omega)^{2} \Omega^{4} \\
-\Omega^{2} d \Omega \Omega^{4}-d \Omega \Omega^{6}+\Omega^{8}, \\
\xi_{4}=S\left(\Theta^{4}\right)=S\left[(d \Omega)^{4}-4(d \Omega)^{3} \Omega^{2}+4(d \Omega)^{2} \Omega^{4}\right. \\
\left.+2 d \Omega \Omega^{2} d \Omega \Omega^{2}-4 d \Omega \Omega^{6}\right] .
\end{gathered}
$$

We observe the relations

$$
\begin{aligned}
& d\left[(d \Omega)^{3} \Omega\right]=(d \Omega)^{4}, \\
& d\left[(d \Omega)^{2} \Omega^{3}\right]=(d \Omega)^{3} \Omega^{2}-(d \Omega)^{2} \Omega d \Omega \Omega+(d \Omega)^{2} \Omega^{2} d \Omega, \\
& d\left[d \Omega \Omega^{2} d \Omega \Omega\right]=(d \Omega)^{2} \Omega d \Omega \Omega-d \Omega \Omega(d \Omega)^{2} \Omega+d \Omega \Omega^{2}(d \Omega)^{2}, \\
& d\left[d \Omega \Omega^{5}\right]=(d \Omega)^{2} \Omega^{4}-d \Omega \Omega d \Omega \Omega^{3}+d \Omega \Omega^{2} d \Omega \Omega^{2}-d \Omega \Omega^{3} d \Omega \Omega+d \Omega \Omega^{4} d \Omega, \\
& d\left[\Omega^{7}\right]=d \Omega \Omega^{6}-\Omega d \Omega \Omega^{5}+\cdots+\Omega^{6} d \Omega,
\end{aligned}
$$


from which we deduce

$$
\begin{aligned}
& d S\left[(d \Omega)^{3} \Omega\right]=S\left[(d \Omega)^{4}\right], \\
& d S\left[(d \Omega)^{2} \Omega^{3}\right]=S\left[2(d \Omega)^{3} \Omega^{2}-(d \Omega)^{2} \Omega d \Omega \Omega\right], \\
& d S\left[d \Omega \Omega^{2} d \Omega \Omega\right]=S\left[(d \Omega)^{3} \Omega^{2}+2(d \Omega)^{2} \Omega d \Omega \Omega\right], \\
& d S\left[d \Omega \Omega^{5}\right]=S\left[2(d \Omega)^{2} \Omega^{4}+d \Omega \Omega^{2} d \Omega \Omega^{2}\right], \\
& d S\left[\Omega^{7}\right]=7 S\left[d \Omega \Omega^{6}\right] .
\end{aligned}
$$

From all of these relations we now obtain

$$
\xi_{4}=d S \Phi_{4}
$$

where

$(11.6) \Phi_{4}=(d \Omega)^{3} \Omega-(4 / 5)\left[2(d \Omega)^{2} \Omega^{3}+d \Omega \Omega^{2} d \Omega \Omega\right]+2 d \Omega \Omega^{5}-(4 / 7) \Omega^{7}$.

12. Final solution. The general result that $\xi_{r}$ defines a cohomology class independent of the given connection has been obtained by $\mathrm{A}$. Weil in a more inclusive theorem [4, p.57]. We shall present Weil's proof for our case in an operational form which has certain points of interest in itself.

First we need some remarks on transformations and spaces. We previously defined the space $\mathcal{J}_{q}=\mathfrak{J}_{q}^{0}$ of $q$-forms and now we introduce the Grassmann ring

$$
g=\sum_{q=0}^{n} \oplus \mathcal{F}_{q}
$$

of all differential forms on $M$. This ring has an involution $\omega \rightarrow \omega^{\prime}$ which is defined for $\omega \in \mathcal{F}_{q}$ by $\omega^{\prime}=(-1)^{q} \omega$. The operation of exterior differentiation is then a semi-derivation in the sense that

$$
d(\omega \eta)=d \omega \eta+\omega^{\prime} d \eta
$$

Now let us consider the tangent space $J_{0}^{1}$. This is a linear space over the ring $\mathcal{F}=\mathcal{F}_{0}$ of functions. If we extend the ring of scalars to $\mathcal{G}$ we obtain the space

$$
d=G \otimes r_{0}^{1}=\sum_{q=0}^{n} \oplus r_{q}^{1},
$$


which may now be considered as a linear space over the ring with involution $C_{\text {. }}$ A linear transformation $\mathbf{C}$ is a mapping on $f f$ into of such that

$$
\mathbf{C}(\mathbf{v}+\mathbf{w})=\mathbf{C}(\mathbf{v})+\mathbf{C}(\mathbf{w}), \quad \mathbf{C}(\omega \mathbf{v})=\omega \mathbf{C}(\mathbf{v})
$$

for $\mathbf{v}, \mathbf{w} \in \mathcal{L}, \omega \in \mathcal{G}$. A semi-linear transformation $\mathbf{C}$ is a mapping on $\not f$ into $f f$ such that

$$
\mathbf{C}(\mathbf{v}+\mathbf{w})=\mathbf{C}(\mathbf{v})+\mathbf{C}(\mathbf{w}), \quad \mathbf{C}(\omega \mathbf{v})=\omega^{\prime} \mathbf{C}(\mathbf{v})
$$

In either case, the trace $S(\mathbf{C})$ is perfectly well defined and may be computed as usual by the rule

$$
S(\mathbf{C})=S(C), \quad \mathbf{C e}=C \mathbf{e},
$$

where $C$ is a matrix with elements in $G$. Our extension theorem for linear transformations says in part that a linear transformation $\mathbf{B}$ over $\mathcal{J}^{\prime}$ on $\gamma_{0}^{1}$ to $g_{1}^{1}$ may

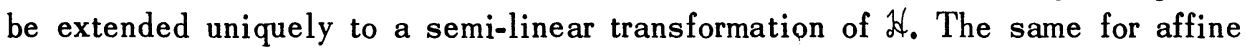
connections says that an affine connection $\mathbf{d}$ on $\zeta_{0}^{1}$ to $\zeta_{1}^{1}$ may be extended uniquely to a semi-derivation $\mathbf{d}$ of $\not d$, this latter signifying that

$$
\mathbf{d}(\omega \mathbf{v})=d \omega \mathbf{v}+\omega^{\prime} \mathbf{d v} \text {. }
$$

We note the following: If $\mathbf{d}$ and $\overline{\mathbf{d}}$ are affine connections, then $\mathbf{d}^{2}$ and $\mathbf{d} \overline{\mathbf{d}}+\overline{\mathbf{d} d}$ are linear on ff. If $_{\mathbf{B}_{1}}$ and $\mathbf{B}_{2}$ are semi-linear on $\mathcal{H}_{\text {, then }} \mathbf{B}_{1} \mathbf{B}_{2}$ is linear. If $\mathbf{d}$ is an affine connection and $B$ is semi-linear on $f f$, then $\mathbf{d B}+\mathbf{B d}$ is linear. This last is proved by first observing that

$$
(d \omega)^{\prime}=-d \omega^{\prime}
$$

and making the computation

$$
\begin{aligned}
(\mathbf{d B}+\mathbf{B d})(\omega \mathbf{v}) & =\mathbf{d}\left(\omega^{\prime} \mathbf{B v}\right)+\mathbf{B}\left(d \omega \mathbf{v}+\omega^{\prime} \mathbf{d v}\right) \\
& =d \omega^{\prime} \mathbf{B v}+\omega \mathbf{d B v}-d \omega^{\prime} \mathbf{B v}+\omega \mathbf{B d v}=\omega(\mathbf{d B}+\mathbf{B d}) \mathbf{v}
\end{aligned}
$$

We want to show that if $\mathbf{d}$ and $\overline{\mathbf{d}}$ are affine connections, then, for each $k$, $S\left(\overline{\mathbf{d}}^{2 k}\right)-S\left(\mathbf{d}^{2 k}\right)$ is an exact differential form. As usual, we set $\overline{\mathbf{d}}=\mathbf{d}+\mathbf{B}$ and we have:

Lemma 12.1. $d S\left(\mathbf{d}^{2 k} \mathbf{B}\right)=S\left[\mathbf{d}^{2 k}(\mathbf{d B}+\mathbf{B d})\right]$.

Proof. We have 


$$
\left(\mathbf{d}^{2 k} \mathbf{B}\right) \mathbf{e}=\mathbf{d}^{2 k}(B \mathbf{e})=B \Theta^{2 k} \mathbf{e}, S\left(\mathbf{d}^{2 k} \mathbf{B}\right)=S\left(B \Theta^{2 k}\right)
$$

hence

$$
\begin{aligned}
d S\left(\mathbf{d}^{2 k} \mathbf{B}\right)=S\left[d\left(B \Theta^{2 k}\right)\right] & =S\left[d B \Theta^{2 k}-B\left(\Omega \Theta^{2 k}-\Theta^{2 k} \Omega\right)\right] \\
& =S\left[(d B-B \Omega-\Omega B) \Theta^{2 k}\right] .
\end{aligned}
$$

On the other hand,

$$
(\mathbf{d B}+\mathbf{B d}) \mathbf{e}=\mathbf{d}(B \mathbf{e})+B(\Omega \mathbf{e})=(d B-B \Omega-\Omega B) \mathbf{e},
$$

which gives the result.

Now we follow the device of Weil in considering the linear series of affine connections

$$
\mathbf{d}_{t}=\mathbf{d}+t \mathbf{B}, \quad 0 \leq t \leq 1
$$

which leads from $\mathbf{d}=\mathbf{d}_{0}$ to $\overline{\mathbf{d}}=\mathbf{d}_{1}$. We hold $\mathbf{B}$ fixed and replace $\mathbf{d}$ by $\mathbf{d}_{t}$ in the lemma to obtain:

Corollary 12.2. $d S\left(\mathbf{d}_{t}^{2 k} \mathbf{B}\right)=S\left\{\mathbf{d}_{t}^{2 k}\left[(\mathbf{d B}+\mathbf{B d})+2 t \mathbf{B}^{2}\right]\right\}$.

On replacing $\mathbf{d}_{t}$ by $\mathbf{d}+t \mathbf{B}$ and expanding, we see that the right side of the last formula becomes a polynomial in $t$ with coefficients traces of certain operators. Such polynomials may be manipulated formally in order to derive relations. One must, of course, pay attention to the noncommutativity of products of operators. In this way we may write

$$
\begin{aligned}
& (\partial / \partial t) \mathbf{d}_{t}^{2}=(\partial / \partial t)\left(\mathbf{d}^{2}+t(\mathbf{d B}+\mathbf{B d})+t^{2} \mathbf{B}^{2}\right)=(\mathbf{d B}+\mathbf{B d})+2 t \mathbf{B}^{2}, \\
& (\partial / \partial t) \mathbf{d}_{t}^{2 k+2}=\left[(\partial / \partial t) \mathbf{d}_{t}^{2}\right] \mathbf{d}_{t}^{2 k}+\mathbf{d}_{t}^{2}\left[(\partial / \partial t) \mathbf{d}_{t}^{2}\right] \mathbf{d}_{t}^{2 k-2}+\cdots ;
\end{aligned}
$$

hence

$$
(\partial / \partial t) S\left(\mathbf{d}_{t}^{2 k+2}\right)=(k+1) S\left\{\mathbf{d}_{t}^{2 k}\left[(\mathbf{d B}+\mathbf{B d})+2 t \mathbf{B}^{2}\right]\right\}
$$

which gives us:

Cor OLla Ry 12.3. $(\partial / \partial t) S\left(\mathbf{d}_{t}^{2 k+2}\right)=(k+1) d S\left(\mathbf{d}_{t}^{2 k} \mathbf{B}\right)$. 
One now integrates out $t$ in these polynomials to obtain

$$
S\left(\overline{\mathbf{d}}^{2 k+2}\right)-S\left(\mathbf{d}^{2 k+2}\right)=d\left[(k+1) S\left(\int_{0}^{1} \mathbf{d}_{t}^{2 k} \mathbf{B} \partial t\right)\right] .
$$

THEOREM 12.4. The cohomology class $S\left(\Theta^{k}\right)=\xi_{k}$ is independent of the affine connection for each $k=1,2, \ldots$.

These manipulations lead to certain invariants of a pair of affine connections $\mathbf{d}, \overline{\mathbf{d}}=\mathbf{d}+\mathbf{B}$. Let us write

$$
\overline{\mathbf{d}}^{2 k}=(\mathbf{d}+\mathbf{B})^{2 k}=\mathbf{d}^{2 k}+\sum_{j=1}^{2 k-1} F_{2 k, j}(\mathbf{d}, \mathbf{B})+\mathbf{B}^{2 k} .
$$

Thus $F_{2 k, j}(\mathbf{d}, \mathbf{B})$ is the sum of all monomials in $\mathbf{d}$ and $\mathbf{B}$ in which $\mathbf{B}$ appears $j$ times. Since $S\left(\mathbf{B}^{2 k}\right)=0$ we have

$$
S\left(\overline{\mathbf{d}}^{2 k}\right)=S\left(\mathbf{d}^{2 k}\right)+\sum_{j=1}^{2 k-1} S\left[F_{2 k, j}(\mathbf{d}, \mathbf{B})\right]
$$

The theorem tells us that the sum on the right side is an exact differential. If for convenience we also set

$$
F_{2 k, 0}(\mathbf{d}, \mathbf{B})=\mathbf{d}^{2 k} \quad \text { and } \quad F_{2 k, 2 k}(\mathbf{d}, \mathbf{B})=\mathbf{B}^{2 k}
$$

then we have:

THЕ OR м 12.5. For each $k=1,2, \cdots$, and $j=1,2, \cdots, 2 k-1$, we have

$$
S\left[F_{2 k, j}(\mathbf{d}, \mathbf{B})\right]=d S\left[(k / j) F_{2 k-2, j-1}(\mathbf{d}, \mathbf{B}) \mathbf{B}\right] .
$$

Proof. By Formula (12.1) we have

$$
\begin{aligned}
& \sum_{j=1}^{2 k-1} S\left[F_{2 k, j}(\mathbf{d}, \mathbf{B})\right]=\mathbf{d}\left[k S\left(\int_{0}^{1} \mathbf{d}_{t}^{2 k-2} \mathbf{B} \partial t\right)\right] \\
& \quad=d S\left[k \int_{0}^{1} \sum_{j=0}^{2 k-2} F_{2 k-2, j}(\mathbf{d}, \mathbf{B}) \mathbf{B} t^{j} \partial t\right]=d\left\{\sum_{j=0}^{2 k-2}(k /(j+1)) S\left[F_{2 k-2, j}(\mathbf{d}, \mathbf{B}) \mathbf{B}\right]\right\}
\end{aligned}
$$




$$
\sum_{j=1}^{2 k-1} S\left[F_{2 k, j}(\mathbf{d}, \mathbf{B})\right]=\sum_{j=1}^{2 k-1} d S\left[(k / j) F_{2 k-2, j-1}(\mathbf{d}, \mathbf{B}) \mathbf{B}\right] .
$$

One replaces $\mathbf{B}$ by $t \mathbf{B}$ in this relation and equates coefficients of the various powers of $t$ to obtain the desired result.

It could have been mentioned earlier that each $F_{2 k, j}(\mathbf{d}, \mathbf{B})$ is a linear transformation on $\mathcal{H}$. This can be seen by writing

$$
\mathbf{d}_{t}^{2 k}-\mathbf{d}^{2 k}=\sum_{j=1}^{2 k} F_{2 k, j}(\mathbf{d}, \mathbf{B}) t^{j}
$$

and observing that the linearity of the left side implies that of the right. Again one "equates coefficients" to obtain the linearity of the individual terms.

Here is a problem suggested by the above investigations. Let $A$ be a nonsingular $n$-by- $n$ matrix of functions, $\Delta=d A A^{-1}$, and

$$
|t I-A|=t^{n}-a_{1} t^{n-1}+\cdots \pm a_{n}
$$

One easily verifies the relations

$$
\begin{aligned}
& d\left(\Delta^{2 r-1}\right)=\Delta^{2 r}, d\left(\Delta^{2 r}\right)=0, \\
& d\left(\Delta^{2 r} A\right) A^{-1}=\Delta^{2 r+1}, S\left(\Delta^{2 r}\right)=0 .
\end{aligned}
$$

We also have

$$
S(\Delta)=d a_{n} / a_{n}
$$

The problem is to find expressions for the traces $S\left(\Delta^{2 r-1}\right)$ of odd powers of $\Delta$ in terms of the invariants $a_{1}, \ldots, a_{n}$.

13. Projective invariants. We now return to some of our earlier considerations. In this section, $\sigma_{0}$ will denote a fixed one-form, $\mathbf{H}$ the transformation of (10.10), $\mathbf{H}^{*}$ its adjoint, as given in Lemma 10.4 , and $\mathbf{B}=\mathbf{H}+\mathbf{H}^{*}$, so that

$$
\mathbf{B v}=\sigma_{0} \mathbf{v}+\mathbf{C}\left(\sigma_{0} \mathbf{v}\right) d P
$$

where $\mathbf{v}$ denotes, as it always shall in this section, an element of $g_{0}^{1}$. According to $(10.18)$ we have 


$$
\mathbf{B}^{2} \mathbf{v}=-\mathbf{C}\left(\sigma_{0} \mathbf{v}\right) \sigma_{0} d P
$$

We now let $\mathbf{d}$ denote a symmetric connection and shall prove the formulas

$$
\begin{aligned}
& \mathbf{d B v}=d \sigma_{0} \mathbf{v}-\sigma_{0} \mathbf{d v}+d \mathbf{C}\left(\sigma_{0} \mathbf{v}\right) d P \\
& \mathbf{B d v}=\sigma_{0} \mathbf{d v}+\mathbf{C}\left(\sigma_{0} \mathbf{d v}\right) d P-\mathbf{C}(\mathbf{d v}) \sigma_{0} d P .
\end{aligned}
$$

The first is evident since

$$
\mathbf{d}(d P)=\mathbf{d}^{2} P=0
$$

To prove the second, one notes that each member is linear in $d v$, so that it suffices to prove the analogous formula in which $d v$ is replaced by an arbitrary element of $\mathfrak{J}_{1}^{1}$, or, indeed, by a generator. Thus let

$$
\mathbf{z}=\lambda \mathbf{w} \in \mathfrak{J}_{1}^{1} \text {, where } \lambda \in \mathcal{J}_{1}^{0}, w \in \mathfrak{J}_{0}^{1} .
$$

Then

$$
\begin{aligned}
\mathbf{B z} & =\mathbf{B}(\lambda \mathbf{w})=-\lambda \mathbf{B w}=-\lambda \sigma_{0} \mathbf{w}-\lambda \mathbf{C}\left(\sigma_{0} \mathbf{w}\right) d P \\
& =\sigma_{0} \mathbf{z}-\left[\mathbf{C}\left(\lambda \sigma_{0} \mathbf{w}\right)+\mathbf{C}(\lambda \mathbf{w}) \sigma_{0}\right] d P=\sigma_{0} \mathbf{z}+\mathbf{C}\left(\sigma_{0} \mathbf{z}\right) d P-\mathbf{C}(\mathbf{z}) d P .
\end{aligned}
$$

Having this, we form the new connection $\overline{\mathbf{d}}=\mathbf{d}+\mathbf{B}$, also symmetric, and have

$$
\bar{d}^{2}=d^{2}+d B+B d+B^{2},
$$

so that

$$
\overline{\mathbf{d}}^{2} \mathbf{v}=\mathbf{d}^{2} \mathbf{v}+d \sigma_{0} \mathbf{v}+\left[d \mathbf{C}\left(\sigma_{0} \mathbf{v}\right)+\mathbf{C}\left(\sigma_{0} \mathbf{d} \mathbf{v}\right)-\mathbf{C}(\mathbf{d v}) \sigma_{0}-\mathbf{C}\left(\sigma_{0} \mathbf{v}\right) \sigma_{0}\right] d P
$$

To proceed, we need the following result:

LEMM A 13.1. If $\lambda$ is a one-form, then $\mathbf{C}(\lambda d P)=(n-1) \lambda$.

For

$$
\mathbf{C}(\lambda d P)=\mathbf{C}\left(\lambda \sigma^{i} \mathbf{e}_{i}\right)=\mathbf{C}\left(\sigma^{i} \mathbf{e}_{i}\right) \lambda-\mathbf{C}\left(\lambda \mathbf{e}_{i}\right) \sigma^{i}=n \lambda-\lambda=(n-1) \lambda .
$$

It now follows that (13.4) implies the following formula:

$$
\overline{\mathbf{d}}^{2} \mathbf{v}-\frac{1}{n-1} \mathbf{C}\left(\overline{\mathbf{d}}^{2} \mathbf{v}\right) d P
$$




$$
=\mathbf{d}^{2} \mathbf{v}-\frac{1}{n-1} \mathbf{C}\left(\mathbf{d}^{2} \mathbf{v}\right) d P+d \sigma_{0} \mathbf{v}-\frac{1}{n-1} \mathbf{C}\left(d \sigma_{0} \mathbf{v}\right) d P
$$

According to (10.17) we have

$$
\bar{\xi}=\xi(\overline{\mathbf{d}})=\xi+(n+1) d \sigma_{0}
$$

hence

$$
\begin{aligned}
\frac{1}{n+1} \bar{\xi} \mathbf{v} & =\frac{1}{n+1} \xi \mathbf{v}+d \sigma_{0} \mathbf{v}, \\
& \frac{1}{n^{2}-1} \mathbf{C}(\bar{\xi} \mathbf{v})=\frac{1}{n^{2}-1} \mathbf{C}(\xi \mathbf{v})+\frac{1}{n-1} \mathbf{C}\left(d \sigma_{0} \mathbf{v}\right) .
\end{aligned}
$$

Combining (13.5) and (13.7) yields

$$
\begin{aligned}
\overline{\mathbf{d}}^{2} \mathbf{v}-\frac{1}{n-1} \mathbf{C}\left(\overline{\mathbf{d}}^{2} \mathbf{v}\right) d P-\frac{1}{n+1} \bar{\xi} \mathbf{v}+\frac{1}{n^{2}-1} \mathbf{C}(\bar{\xi} \mathbf{v}) d P \\
=\mathbf{d}^{2} \mathbf{v}-\frac{1}{n-1} \mathbf{C}\left(\mathbf{d}^{2} \mathbf{v}\right) d P-\frac{1}{n+1} \xi \mathbf{v}+\frac{1}{n^{2}-1} \mathbf{C}(\xi \mathbf{v}) d P .
\end{aligned}
$$

The operator

$$
\mathbf{v} \rightarrow \mathbf{d}^{2} \mathbf{v}-\frac{1}{n-1} \mathbf{C}\left(\mathbf{d}^{2} \mathbf{v}\right) d P-\frac{1}{n+1} \xi \mathbf{v}+\frac{1}{n^{2}-1} \mathbf{C}(\xi \mathbf{v}) d P
$$

defined on $\gamma_{0}^{1}$ to $\gamma_{2}^{1}$ by means of the given connection $d$ is the well-known Weyl projective curvature tensor $[6, \mathrm{p} .88]$ expressed in operator form. The equation (13.8) states that it is invariant under a projective change of connection.

Another invariant of this type can be obtained for the changes of connection which preserve parallelism. Let $\mathbf{d}$ be an arbitrary connection, $\mathbf{H v}=\sigma_{0} \mathbf{v}$ as above, and note

$$
\begin{aligned}
& \mathbf{H}^{2} \mathbf{v}=0 \\
& \mathbf{d H v}=d \sigma_{0} \mathbf{v}-\sigma_{0} \mathbf{d v} \mathbf{} \\
& \mathbf{H d v}=\sigma_{0} \mathbf{d v} .
\end{aligned}
$$

Thus if $\overline{\mathbf{d}}=\mathbf{d}+\mathbf{H}$ is a connection for which parallelism has the same meaning 
as for $d$, then

$$
\overline{\mathbf{d}}^{2} \mathbf{v}=\mathbf{d}^{2} \mathbf{v}+d \sigma_{0} \mathbf{v}
$$

By Theorem 10.5, we have $\bar{\xi}=\xi+n d \sigma_{0}$; hence

$$
\overline{\mathbf{d}}^{2} \mathbf{v}-\frac{1}{n} \bar{\xi} \mathbf{v}=\mathbf{d}^{2} \mathbf{v}-\frac{1}{n} \xi \mathbf{v}
$$

This shows that the transformation on $y_{0}^{1}$ to $J_{2}^{1}$ defined by

$$
\mathbf{v} \rightarrow \mathbf{P v}=\mathbf{d}^{2} \mathbf{v}-\frac{1}{n} \xi \mathbf{v}
$$

is invariant under any change of connection which preserves parallelism. Expressed in terms of coordinates, this defines a tensor which may be called the parallel curvature tensor; it is given by

$$
P_{i k l}^{j}=R_{i k l}^{j}-\frac{1}{n} R_{h}{ }^{h} k l \text {. }
$$

To complete this section, we shall also find an invariant tensor for the class of transformations $\mathbf{H}^{*}$. We first have

$$
\mathbf{H}^{*}(d P)=-\sigma_{0} d P
$$

For

$$
\mathbf{H}^{*}(d P)=\mathbf{H}^{*}\left(\sigma^{i} \mathbf{e}_{i}\right)=-\sigma^{i} \mathbf{C}\left(\sigma_{0} \mathbf{e}_{i}\right) d P=-\sigma_{0} d P
$$

It easily follows that for $\overline{\mathbf{d}}=\mathbf{d}+\mathbf{H}^{*}$ we have

$$
\begin{aligned}
& \overline{\mathbf{d}^{2}} P=\mathbf{d}^{2} P-\sigma_{0} d P \\
& \mathbf{C}\left(\overline{\mathbf{d}}^{2} P\right)=\mathbf{C}\left(\mathbf{d}^{2} P\right)-(n-1) \sigma_{0} ;
\end{aligned}
$$

hence

$$
\overline{\mathbf{d}}^{2} P-\frac{1}{n-1} \mathbf{C}\left(\overline{\mathbf{d}}^{2} P\right) d \mathbf{l}^{P}=\mathbf{d}^{2} P-\frac{1}{n-1} \mathbf{C}\left(\mathbf{d}^{2} P\right) d P
$$

A given affine connection d defines an element $\mathbf{z}=\mathbf{z}(\mathbf{d})$ of $T_{2}^{1}$ given by 


$$
\mathbf{z}=\mathbf{d}^{2} P-\frac{1}{n-1} \mathbf{C}\left(\mathbf{d}^{2} P\right) d P
$$

We have shown that $\mathbf{z}$ is unchanged when $\mathbf{d}$ is replaced by $\mathbf{d}+\mathbf{H}^{*}$. To compute the components of the tensor which $\mathbf{z}$ defines, we set

$$
\mathbf{z}=\frac{1}{2} Z_{j k}^{j} \sigma^{j} \sigma^{k} \mathbf{e}_{i}
$$

and have

$$
Z_{j k}^{i}=T_{j k}^{i}-\frac{1}{n-1}\left(T_{j l}^{l} \delta_{k}^{i}-T_{k l}^{l} \delta_{j}^{i}\right)
$$

which is the case because

$$
\begin{aligned}
& \mathbf{d}^{2} P=\frac{1}{2} T^{i}{ }_{j k} \sigma^{j} \sigma^{k} \mathbf{e}_{i}, \quad \mathbf{C}\left(\mathbf{d}^{2} P\right)=T^{l}{ }_{j l} \sigma^{j}, \\
& \mathbf{C}\left(\mathbf{d}^{2} P\right) d P=T^{l}{ }_{j l} \sigma^{j} \sigma^{i} \mathbf{e}_{i}=T^{l}{ }_{j l} \delta_{k}^{i} \sigma^{j} \sigma^{k} \mathbf{e}_{i} \\
&=\frac{1}{2}\left(T^{l}{ }_{j l} \delta_{k}^{i}-T_{k l}^{l} \delta_{j}^{i}\right) \sigma^{j} \sigma^{k} \mathbf{e}_{i} .
\end{aligned}
$$

Here $T$ is the torsion tensor as given in $\S 7$.

\section{REFERENCES}

1. N. Bourbaki, Algèbre multilineaire, Paris, 1948.

2. S.S. Chern, Note on affinely connected manifolds, Bull. Amer. Math. Soc. 53 (1947), 820-823.

3. Correction to my paper "Note on affinely connected manifolds," Bull. Amer. Math. Soc. 54 (1948), 985 - 986.

4. Topics in differential geometry, mimeographed notes, Princeton, 1951. 1950.

5. G. de Rham and K. Kodairr, Harmonic integrals, mimeographed notes, Princeton,

6. L. P. Eisenhart, Non-Riemannian geometry, New York, 1927.

7. H. Flanders, Development of an extended exterior differential calculus, Trans. Amer. Math. Soc. 75 (1953), 311 - 326.

8. H. Samelson, On Chern's invariant for Riemannian 4-manifolds, Proc. Amer. Math. Soc. 1 (1950), 415-417. 



\section{PACIFIC JOURNAL OF MATHEMATICS}

\section{EDITORS}

\author{
H.L. ROYDEN \\ Stanford University \\ Stanford, California \\ E. HEWITT \\ University of Washington \\ Seattle 5, Washington
}

\author{
R. P. DILWORTH \\ California Institute of Technology \\ Pasadena 4, California \\ * Alfred Horn \\ University of California \\ Los Angeles 24, California
}

\section{ASSOCIATE EDITORS}

\section{H, BUSEMANN \\ HERBERT FEDERER}

MARSHALL HALL

\section{P.R. HALMOS \\ HEINZ HOPF}

ALFRED HORN

\author{
R.D. JAMES \\ BØRGE JESSEN \\ PAUL LÉVY
}

GEORGE PÓLYA

J.J. STOKER

KOSAKU YOSIDA

\section{SPONSORS}

UNIVERSITY OF BRITISH COLUMBIA

UNIVERSITY OF SOUTHERN CALIFORNIA

CALIFORNIA INSTITUTE OF TECHNOLOGY

UNIVERSITY OF CALIFORNIA, BER KELEY

STANFORD RESEARCH INSTITUTE

STANFORD UNIVERSITY

UNIVERSITY OF CALIFORNIA, DAVIS

UNIVERSITY OF. UTAH

UNIVERSITY OF CALIFORNIA, LOS ANGELES

WASHINGTON STATE COLLEGE

UNIVERSITY OF CALIFORNIA, SANTA BARBARA

UNIVERSITY OF WASHINGTON

MONTANA STATE UNIVERSITY

UNIVERSITY OF NEVADA

OREGON STATE COLLEGE

AMERICAN MATHEMATICAL SOCIETY

HUGHES AIRCRAFT COMPANY

UNIVERSITY OF OREGON

SHELL DEVELOPMENT COMPANY

\section{UNIVERSTTY OF SOUTHERN CALIFORNIA}

Mathematical papers intended for publication in the Pacific Journal of Mathematics should be typewritten (double spaced), and the author should keep a complete copy. Manuscripts may be sent to any of the editors. Manuscripts intended for the outgoing editors should be sent to their successors. All other communications to the editors should be addressed to the managing editor, Alfred Horn, at the University of California Los Angeles 24, California.

50 reprints of each article are furnished free of charge; additional copies may be obtained at cost in multiples of 50 .

The Pacific Journal of Mathematics is published quarterly, in March, June, September, and December. The price per volume (4 numbers) is $\$ 12.00$; single issues, $\$ 3.50$; back numbers (Volumes $1,2,3$ ) are available at $\$ 2.50$ per copy. Special price to individual faculty members of supporting institutions and to individual members of the American Mathematical Society: $\$ 4.00$ per volume; single issues, $\$ 1.25$.

Subscriptions, orders for back numbers, and changes of address should be sent to the publishers, University of California Press, Berkeley 4, California.

Printed at Ann Arbor, Michigan. Entered as second class matter at the Post Office, Berkeley, California.

* During the absence of E.G. Straus.

UNIVERSITY OF CALIFORNIA PRESS - BERKELEY AND LOS ANGELES 


\section{Pacific Journal of Mathematics}

\section{Vol. 5, No. $3 \quad$ November, 1955}

Nesmith Cornett Ankeny and S. Chowla, On the divisibility of the class number of quadratic fields ............................. 321

Cecil Edmund Burgess, Collections and sequences of continua in the plane ........................................ 325

Jane Smiley Cronin Scanlon, The Dirichlet problem for nonlinear elliptic equations....................................... 335

Arieh Dvoretzky, A converse of Helly's theorem on convex sets ......... 345

Branko Grünbaum, On a theorem of L. A. Santaló................ 351

Moshe Shimrat, Simple proof of a theorem of P. Kirchberger .......... 361

Michael Oser Rabin, A note on Helly's theorem . ................... 363

Robert E. Edwards, On factor functions . . ................... 367

Robert E. Edwards, On certain algebras of measures ............... 379

Harley M. Flanders, Methods in affine connection theory.............. 391

Alfred Huber, The reflection principle for polyharmonic functions ........ 433

Geoffrey Stuart Stephen Ludford, Generalised Riemann invariants ....... 441

Ralph Gordon Selfridge, Generalized Walsh transforms............. 451 